\title{
HOWELL'S PACIFIC COAST PLANTS.
}

Price : less than 50, 8 cents each : 50 to 500,5 cents each : 500 or more, 4 cents each. Sent prepaid to any part of the United States.

1 Money-order office, Portland, Oregon.

Anemone deltoidea Hook.

- Thalictrum occidentale Gray. polyearpum Watson.

Myosurus minimus $\mathrm{L}$. aristatus Benth. sessilis Watson.

Ranunculus Flammula L. var. reptans Meyer. glaberrimus Hook.

Hookeri Reg.

Nelsoni v. tenellus Gray. occidentalis Nutt. orthorhynehus Hook. muricatus L.

Caltha leptosepala DC.

Coptis asplenifolia Salisb. oceidentalis T. \& G.

Isopyrum stipitatum Gray.

Aquilegia formosa F. if M.

Delphiniun Menzicsii DC: simplex Dougl. trolliifslium Gray.

Actat spicita $r$. arguta Torr.

Betheris anuifolium Pursh. nervosit Pursh.

Vaneouveria hexanira M. \& I)

Aehlys triphylla DC.

Eschscholtria Califomica (:

Platystignia Oreganum B. \& $\mathrm{H}$.

Diesintra formesa $D C$.

Coryclalis seouleri Hook.

Nasturtium eturvisiliqua Nutt.

Arabis Hollmellii Hornem.

C'usickii Watson.

suffirutescens Watson.

fureatia Wutson.

longirostris IV atson.

Cardamine angulata Hook. hirsuta I.

4 Dentaria tenella Pursh.

12 Draba Caroliniana Walt.

19 Theiranthus Menziesii $\vec{B}$ \& $H$.

44 Platyspermum scapigerum $\mathrm{Hk}$.

45 Nisymbrium linifolium Xutt. eanescens Nutt.

Hartwegianum Fourner

Snelowskia ealyeina Meyer.

Thelypodium laciniatum End.
50 Braya Oregonensis Gray.

51 Capsella divaricata Walp.

52 Lepidium dietyotum Gray.

53

54

55

56

57

58

59

60

61

62

63

64

65

66

67

68

69

70

71

72

73

74

75

76

77

78

79

80

81

82

var, acutidens Griv
Thysanocarpus pusillus Hook eurvipes Hook.

Viola sarmentosa Dougl. glabella Nutt. Hallii Gray. trinervata Howell. canina v. adunca Grav.

Polygala eucullata Benth.

silene Lyallii Watson. Menziesii Hook. Hookeri Nutt. Oregana Watson. Douglasii Hook. Seouleri Hook.

Cerustium nutans Raf. arvense $\mathrm{L}$.

Stellaria crispa C. \& S. borealis Bigel.

Arenaria capillaris Poir. Franklinii Dougl. Donglasii $\mathrm{T}$, \& $\mathrm{G}$. Californica Brewer. Miehauxii Hooker $\mathrm{f}$. verna $v$. hirta Watson. maerophylla Hook.

Sagina oecidentalis Watson. Limnzei Presl.

Claytonia laneeolata Pursh. perfoliata Donn. exigua T.\& $G$. Sibiriea $\mathrm{L}$. diffusa Nutt. bulbifera Gray. Chamissonis Esch. linearis Dougl. dichotoma Nutt.

Montia fontana L. Howellii Watson.

Spraguea umbellata Torr.

Hyperieum Seouleri Hook. anagaloides C. \& S.

Malvastrum Munroanum firty Linum digynum Gray: nicranthum Gray. 
100 Limnanthes Douglasii R. $\mathrm{Br}$.

101 alba Hartw.

102 Florkea proserpinacoides 11 .

103 Oxalis Oregana Nutt.

104 Enonymus occidentalis Nutt.

\05 Pachystima myrsinites Raf.

106 Rhamnus Purshiana DC.

107 Ceanothus sanguineus Pursh.

tos velutinus Dougl.

109 integerrimus H. \& A.

110 prostratus Benth.

111 Acer maerophyllum Pursh.

112

113 glabrum Torr. circinatum Pursh.

114 Rhus diversiloba T. \& G.

115 Thermopsis montana Nutt.

116 Lupinus rivularis Dougl.

117

118

119

120

121

122

123

124

125

126 Trifolium megacephalum Nutt

127 eriocephalum Nutt.

128 plumosum Dougl.

129 longipes Nutt.

130 Maeræi H. \& A.

131 ciliatum Nutt.

132 gracilentum T. \& G.

133 involueratum Willd.

134 tridentatum Lindl.

135 pauciflorum Nutt.

136 mierodon $\mathrm{H}$. \& A.

137 cyathiferum Lindl.

138 Hosiekia crassifolia Benth.

139 bieolor Dougl.

140 parviflora Benth.

141 decumbens Benth.

142 Psoralea physoides Dougl.

143 Astragalus Purshii Dougl.

144 inflexus Dougl.

145 speirocarpus Gray.

146 filipes Torr.

147 Howellii Gray.

148 selerocarpus Gray.

149 drepanolobus Gray.

150 arrectus Gray.

151 collinus Dougl. reventus Gray.

Ientiginosus Dougl
156

succumben: Dougl.

157 Vicia Americana var. truneati

158 Lathyrus littoralis Endl.

159

160

Nevadensis Watson.

161 Prunus mollis Walp.

162 demissa Walp.

163 Nuttallia cerasiformis $T$. \& 6 .

164 Spirea-hetulifolia Pallas.

165

166

167

168

169

170 chamedrifolia C. \& S.

Douglasii Hook. ariæfolia Smith. thmosa Nutt Aruncus $\mathrm{I}$.

occidentalis II itson

171 Neillia opulifolia $\mathrm{v}$. nollis Bre.

172 Rubus Nutkanus Mocino.

173 spectabilis Pursh.

174 lencodernis Dougl.

175 ursinus $(. \&$ \&

176 pedatus smitl.

17T Purshia tridentata $\mathrm{DC}$.

178 Cereoearpus farvifolius Nutt.

179 Geum macrophyllum Willd.

180 Potentilla gracilis Dungl.

181 rivalis $x$. millegraua $W_{\text {at }}$

182 gelida Neyer.

18:; Anserina v. grandis lech.

184 Horkelia fusea Lindl.

185 eongesta Hook.

186 Alchemilla oceidentalis Nutt.

197 Rosa Nutkana Presl.

188 gymnocarpa Nutt.

189 Pirus rivularis Dougrl.

190 sambucifolia C. \&. .

191 Cratrgus Douglasii Lindl.

192 Saxifraga integrifolia Hoek.

193 Nertensiana Bong.

194 bronchialis v. cherleroide-

195 exspitosa L.

196 elegans Nutt.

197 Sullivantia Oregana Watson.

198 Borkinia oceidentalis $T$. \& $\mathrm{f}$.

199 Tolmiea Menziesii T. \& G.

200 Suksdorfia violacea Gray.

201 Tellima grandiflora R. Br.

202

203

parviflora Hook.

204 Tiarella tifoliata

205 unifoliata Hook.

206 Mitella trifida Graham.

207 eaulescens Nutt.

208 pentandra Hook.

209 Breweri Gray.

210 Heucharia micrantha Dougl. 211 glabella Nutt. 
212 Parnassia fimbriata Køenig. 213 Philadelphus Lewisii Pursh. 214 Whipplea modesta Torr. 215 Ribes Lobbii Gray.

-25 Till a angustifolia Nutt.

226 Sedum Oreganum Nutt.

228 Drosera rotundifolia L.

229 Anglica Hudson.

2: 0 Ludwig:a palustris Ell. 231 Fpilobium spicatum Lam.

232

233

234

235

236

237 Gayophyfum diffusum T. \& $\mathrm{G}$.

238 racemosum T. \& $\mathrm{G}$.

239 Boisiluvalia Torreyi Watson.

240 Gorl tia lepi la Lindl.

241 tenella Watson.

242 amena Lilj.

243 Gauma parviltora Dougl.

244 Cirexa Pacifiea Iseh, \& Mag.

245 Crantzia lin sata Nutt.

246 Eryagium potiolitum var.

247 Sanicula Menziesii H. \&.A.

248 Nevarlonsis Watson.

249 bipinnatificla Dougl.

250 Carum Gain tneri B. \& H.

251 Osmo rhiza nuda Torr.

252 Gly osma o vide rtale Nutt.

:53 ambignum Gray.

254 (Enanthe sarmentosa Nutt.

2 ;5 Ligustieum apiifolium B. \& $\mathrm{H}$.

256 Cymopterus terelointhinus

257 Peucedanum leiocarpum Nutt.

$-259$

259 triturnatum Nutt. simplex Nutt. Hallii Watson. u'riculatum Nutt. millifolium Watson.

265 Ferula multifida Gray. 266 Fatsia horrida B. \& H. 267 Cornus Nuttallii Audubon.
268 pubescens Nutt. 269 Garrya Fremontii Torr. 270 Sambucus glauca Nutt. 271 racemosa v. arborescens, 272 Linnæa borealis v. longiflora 273 Symphoriearpus racemosus M. 274 Lonicera ciliosa Poir.

275 involuerata Banks.

276 Kelloggia galioides Torr.

277 Galium Aparine L.

278

279

280

281 var. Vaillantii Koch. Kamtschaticum Steller. trifidum L.

282 Valerianella congesta Lindl.

283 anomala Gray.

284 Grindelia Oregana Gray.

285 hirsutula H. \& A.

286 nana Nutt.

287

288 Aplopappus Hallii Gray.

289 Bloomeri Gray.

290 carthimoides Gray.

291 stenophyllus Gray.

292 Solidago occidentalis Nutt.

293 elongata Nutt.

294 confertiflora DC.

295 humilis v. nana Gray,

296 Sericocarpus rigidus Lindi.

297 Townsendia florifer Gray.

298 Aster radulinus Gray.

299 modestus Lindl.

300 Douglasii Lindl.

301 foliaceus v. apricus Gray.

302 pulchellus Eaton.

303 frondosus T. \& G.

304 canescens Pursh.

305 Erigeron concinnus T. \& G.

306 poliospermus Gray.

307 Chrysopsidis Gray.

308 eompositus Pursh.

309 filifolius Nutt.

310 decumbens. Nutt.

311 inornatus Gray.

312 divergens T. \& $\mathrm{G}$.

313 acres v. debilis Gray.

314 Micropus Californicus F. \& M.

315 Psilocarpus Oreganus Nutt.

316 Antennaria dimorphia T. \& G

317

318

319

320

321 flagellaris Gray ieyeri tiray. Inzuloides T. \& G. Carpathica R. Br. alpina Grertn. dioica Grertn. racemosa Hook. 
324 Attenocaulon bicolor Hook. 325 Franseria Chamissonis Less. 326 Rudbeckia occidentalis Nutt. 327 Balsamorrhiza sagittata Nutt. 328 Helianthus Nuttallii T. \& G. 329 Coreopsis Atkinsoniana Dougl. 330 Blepharipappus scaber Hook. 331 Madia Nuttallii Gray. 332 glomerata Hook. 333 filipes Gray.

334 Hemizonella Durandi Gray. 335 Lagophylla ramosissima Nutt. 336 Layia glandulosa H. \& A. 337 Eriophyllum caspitosum Dgl. 338 Hulsea nana Gray.

339 Rigiopappus leptoeladus Gray. 340 Chrenactis Douglasii H. \& A.

341 Petasites palmata 6 ray.

342 Crocidium inulticaule Hook.

343 Tetradymia canescens DC. 344 Senecio triangularis Hook. 345 lugens v, exaltatus (iray. 346 fastigiatus Nutt.

347 Bolanderi Gray.

348 Arnica cordifolia Hook,

$349 \quad$ latifolia Bong.

350 amplexicaulis Nutt.

351 Chamissonis Less.

352 saussurea Americana Eaton.

353 Cnicus remotifolius Gray.

354 Microseris nutans Gray.

355 troximei les Gray.

356 Apargidium boreale T. \& $G$.

357 Hieraeium Seouleri Hook.

358 gracile Hook.

359 albiflorum Hook.

360 Crepis occidentalis Nutt.

361 Troximon glaucum Nutt. 362 apargioides Less.

363 heterophyllum Greene.

364 Downingia elegans Torr.

365 Howellia aquatalis Gray.

366 Githopsis specularioides Nutt.

367 Campanula scabrella Engelm.

368 Scouleri Hook.

369 Heteroeodon rariflorum $\mathrm{Nutt}$.

370 Vaccinium occidentale Gray.

371 exspitosum $v$, arbuscula.

372 Myrtillus v. mierophyllum

373 myrtilloides Hook.

374 parvifolium Smith.

375 ovatum Pursh.

376 Aretostaphylos tomentosa. Dgl.

377 Gaultheria shallon Pursh.

378 Kalmia glauea Ait.

379 Menziesia glabella Gray.
380 Rhododendron albiflorim Hk.

381 occidentale Gray.

382 Californicum Hook.

383 Pyrola rotundifolia v. bracteat:

384 picta Smith.

385 Pterospora andromedea Nutt.

386 Sareodes sanguinea Torr.

387 Monotropa Hype pitys L.

388 Trientalis Europaea v. latifolia.

389 Glaux maritima L.

390 Apocynum eannabinum L.

391 Erythrea Douglasii Gray.

392 Gentiana sceptrum Griseb.

393 Frasera nitida Benth.

394 Phlox Douglasii Hook.

395

396

397

398 filia grandiflora Gray

399 linemis Giray.

400 gracilis Hook.

401 Sessei Din.

402 glutinosa Nutt.

403 liniflora v. pha naceiortes

404 Bolanderi Gray.

40.5 tenella Benth.

406 intertexta Steud.

407 aggregata Spreng.

408 Larseni Gray.

409 inconspicua Dougl.

410 Polemonium humile Willd.

411 caruleum L. ?.

412 mieranthum Benth.

413 Hydrophyllum capitatum Dgl.

414 Nemophila parviflora Dougl.

415 Menziesii H. \& A.

416 Phacelia ramosissima Douxl.

417 procera Gray.

418 Menziesii Torr.

419 Romanzoffia Sitchensis Bong.

420 Hesperochiron pumilus Porter.

421 Eriodictyon glutinosum Benth

422 Pectocarya pusilla Gray.

42* Cynoglossum grande Dougl.

424 Echinospermum Redowskii.

425 hispidum.

426 Eritrichium Californieum DC.

427 Seouleri A. DC.

428 fulvum A. DC.

429 tenellum Gray.

430 pterocaryum Torr.

431 glomeratum DC.

432 ambiguum.

433 Amsinckia lycopsoides var.

434 Lithospermum pilosum Nutt.

435
Californicum Gray. 
436 Convolvulus Californicus Ch.

437 Collinsia grandiflora Dougl.

438

4:9

440

441

442

443 Tonella collinsioides Nutt.

444 Chelone nemorosa Dougl:

445 Pentstemon Menziesii Hook.

446

447

448

449

450

451 Mimulus nanus H. \& A

452

453

454

455

456

457

458

459 Gratiola ebracteata Benth.

460 Limosella aquatica $\mathrm{L}$.

461 Synthyris rotundifolia Gray.

462 reniformis Bentl.

463 Veronica Americana Schwein.

464

465 Castilleia parviftora Bong.

466 miniata Dougl.

467 pallida v. septentrionalis

468 Orthorarpus pilosus Watson.

469 attenuatus Gray.

470 (astilleioides Benth.

471 liracteosus Benth.

472 pusillus Benth.

473 hispislus Benth.

474 Corlylanthus eapitatus Nutt.

475 pedicularis (iroenlandica Ritz.

476 racemosa Dougl.

477 bracteosa Benth.

478. Aphyllon faseieulatum Gray.

479 Trichostema oblongum Benth.

180 lanceolatum Centh-

4\$1 Monardella odoratissima Bent.

i82 Palmeri Gray. ?.

483 Mieromeria Douglasii Benth.

184 Audibertia ineana Benth.

485 Lophanthus urticifolius Benth

486 Seutellaria lateriflora $L$.

487 antirrhinoides Benth.

489 Physostegia parviflora Nutt,

489 Stachys palustris $\mathrm{L}$.

490 ciliata v. pubens Gray.

491 Plantago Patatgonica Jacy.
492 Abronia latifolia Esch.

493 Rumex venosus Pursh.

494 occidentalis Watson.

495 Polygonum paronychia C. \& S.

496 erectum L.

497 minimum Watson.

498 coaretatum Dougl.

499 polygaloidos Meisner.

500 imbricatum Nutt.

501 intermedium Nutt.

502 Bistorta L.

503 Davisiæ Brewer.

504 Parryi Greene.

505 Eriogonum pyrolæfolium var.

506 sphærocephalum Dougl.

507 umbellatum Torr.

508 heracleoides Nutt.

509 dichotomum Dougl.

$510 \quad$ nudum Dougl.

511 vimineum Dougl.

512 Sarcobatus rermiculatus Torr.

513 Monolepis chenopodioides $\mathrm{Mo}$.

514 Grayia polygaloides H, \& A.

515 Umbellularia Californiea Nutt.

516 Urtica dioica L.

517 Eremocarpus setigerus Benth.

518 Euphorbia glyptosperma Enge

519 Betula oceidentalis Hook.

520 Alnus rubra Bong.

521 Myrica Californica Cham.

522 Salix lasiandra $v$. lancifolia.

523 longifolia Muhl.

524 sessilifolia Nutt.

525

526

527

528

529

amygdaloides Anders.

530 Quercus Garryana Dougl.

531 chrysolepis Liebm.

532 Asarum caudatum Lindl.

533 Taxus brevifolia Nutt.

534 Juniperis oceidentalis Hook.

535 Thuya gigantea Nutt.

536 Cupressus Nutkaensis.

537 Libocedrus decurrens Torr.

538 Corallorhiza Mertensiana Bong

539 striata Lindl.

540 Calypso borealis Salisb.

541 Habenaria Unalaschensis Wat.

.542 leueostachys Watson.

543 sparsiflora Watson,

544 graeilis Watson.

545 Goodyearia Menziesii Lindl.

546 Listera cordata R. Br.

547 Epipactis gigantea Dougl. 
548 Cypripedium montanum Douy 549 Californicum Gray.

5.50 Iris macrosiphon Torr.

551 tenax Dougl.

552 longipetala Herbert.

553 sisyrinchium bellum Watson.

554 grandiflorum Dougl.

555 Allium schœnoprasum L.

556 madidum Watson.

557 acuminatum Hook.

558 attenuifolium Kell.

559 Brodiæa congesta Smith.

560 Douglasii Watson.

561 laetea Watson.

562 Howellii Watson.

563 Camassia esculenta Lindl.

564 Smilacina racemosa Desf.

565 sessilifolia Nutt.

566 Lilium Columbianum Hanson.

567 Fritillaria lanceolata Pursh.

568 pudica Spreng.

569 Erythronium grandiflorum Pu.

570 giganteum Lindl.

571 Calochortus elegans Pursh.

572 Tolmiei H. \& A.

573 lilacinus Kell.

574 Prosartes Menziesii Don.

575 Oregana Watson.

576 Clintonia uniflora Kunth.

577 Scoliopus Hallii Watson.

578 Trillium ovatum Pursh.

579 Stenanthium occidentale Gray.

$580 \mathrm{Z}$ ygadenus venenosus Watson.

581 Ruppia maritima L.

582 Potamogeton natans L.

583 Triglochin maritimum L.

584 Luzula parviflora $v$. pallida.

585 comosa Meyer.

586 Juncus Balticus Dethard.

587 effusus L.

$588 \quad$ Parryi Engelm.

589 tenuis v. congestus Eng.

590 Leseurii Bol.

591 falcatus Meyer.

592 dubius Engelm.

593 xiphoides Meyer.

594 Cyperus occidentalis Torr.

595 Scirpus pungens Vahl.

596 Eleocharis obtusa Schult.

597 Carex Douglasii Boott.
604

605

606

607

608

609

610

611

612

613

614

615

616 Beckmannia erucæformis Host.

617 Panicum scoparium Lam.

618 Phalaris arundinacea $\mathrm{L}$.

619 Hierochloa borealis R. \& S.

620 maerophylla Thurb.

621 Alopecurus aristulatus Mx.

622 saccatus Vasey.

623 Stipa comata Trin.

624 viridula Trin.

625 Phleum alyinum L.

626 Coleanthus subtilis Seid.

627 Sporobolus gracillimus Vasey.

628 Agrostis foliosa Vasey.

629 seabra Willd.

630 Scouleri Trin.

631 varians Trin.

632 Polypogon littoralis Smith.

633 Cinna pendula Trin.

63 \& Deyeuxia Aleutica Vasey.

635 Canadensis Beauv.

636 Howellii Vasey.

637 Deschampsia exspitosa Beauv.

638 calycina Presl.

639 elongata Munro.

640 latifolia Vasey.

641 Trisetum canescens Buckley.

642 subspicatum v. molle (ir.

643 Avena fatua $L$.

644 Danthonia Californica Bol.

645 Køleria cristata Pers.

646 Eragrostis reptans Nees.

647 Melica bromoides Bol.

648 bulbosa Geyer.

649 Harfordii Bol.

650 Distichlis maritima Raf.

651 Briza minor L.

652 Poa abreviata R. Br.

653 annua L.

654 alpina v, purpurascens.

655 Bolanderi Vasey.

656 Douglasii Nees.

657 gracillima Vesey.

658 pulchella Vasey.

659 serotina Ehrh. 
$\begin{array}{ll}600 & \text { stenantha Trin. } \\ 661 & \text { tenuifolia Nutt. }\end{array}$

662 Glyceria nervata Trin.

663 paueiflora Presl.

664 Festuca microstachys Nutt.

665 ovina $\mathrm{L}$. var.

666 var. polyphylla Vas.

667 rubra v. fallax Hack.

668 tenella Willd.

669 , scabrella Torr.

670 Hordeum pratense Huds.

671 Elymus Sibiricus L.

672 triticoides Nutt.

673 dasystachys Trin.

674 Botrychium ternatum Swartz.

675 Polypodium falcatum Kell.

676 Scouleri Hook. \& Grev.

677 Gymnogramme triangularis $\mathrm{Kf}$

678 Cheilanthes gracillima Eaton.

679 Pellæa densa Hook.

680 Cryptogramine acrostichoides.

681 Pteris aquilina v. lanuginosa.

682 Lomaria spicant Desv.

683 var. serratum Woll.

684 Phegopteris Dryopteris Fee.

685 Aspidium munitum $\mathrm{Kf}$.

686 lonchitis Swarte.

687 rigidum v. argutum Eat.

688 spinulosum v. dilatatum.

689 Cystopteris fragilis Bern.

690 Woodsia Oregana Eaton.

691 Lyeopodium annotinum L.

692 clavatum $\mathrm{J}$.

693 lucidulum Mx.

694 Selaginella rupestris Spring.

695 Douglasii spring.

696 Oregana Eaton.

697 Isoetis nuda Engelm.

698 Howellii Engelm.

699 Marsilia vestita Hook. \& Grev.

T00 Azolla Caroliniana Willel.

\section{MOSSES.}

701 Sphagnum squarrosulum Lesq

702 Dicranoweisia cirrhata Lindb.

703 Dieranum palustre La Pyl.

704 Barbula vinealis Braun.

705 Muelleri B. \& S.

706 Grimmia Scouleri Muell.

707 montana B. \& S.

708 trichophylla Grev.

709 Racomitrium Nevii Watson.

710 lanuginosum Brid.

711 canescens Brid.

712 aciculare Brid.

713 Hedwigia ciliata Ehrh.

714 Braunia californica Lesq.

715 Ptychomitrium Gairdneri Les.

716 Amphoridium Mougeotii Schi.

717 Orthotrichum pulehellum Bru.

718 Lyellii H. \& T.

719 Physcomitrum pyriforme Brid.

720 Bartramia Menziesii Turn.

721 ithyphylla Brid.

722 pomiformis Hedw.

723 fontana Brid,

724 Meesia tristicha B, \& S.

725 Mnium Menziesii Muell.

726 Aulacumnium androgynum.

727 Oligotrichum Lyallii Lindb.

728 Pogonatum alpinum Roehl.

729 Polytrichum piliferum Schreb.

730 Fontinalis Neo-Mexicana.

731 antipyretica L.

732 Dichelyma falcatum Myrin.

733 Alsia abietina Sulliv.

734 Neckera Menziesii Drumm.

735 Douglasii Hook.

736 Antitrichia Californiea Sulliv.

737 eurtipendula v. gigantea.

$7: 8$ Hookeria lucens Smith.

739 Climacium dendroides W.\& M

740 Hypnum erispifolium Hook.

741 lutescens Huds.

742 Nuttallii Wils.

743 megaptilum Sulliv.

744 myosuroides $\mathrm{L}$.

745 stoloniferum Hook.
Stokesii Turn.

Oreganum Sulliv.

Bigelovii Sulliv. uncinatum Hedw. circinale Hook. subimponens Lesq. splendens Hedw. triquetram L. loreum $\mathrm{L}$. 



\section{HOWELL'S PACIFIC COAST PLANTS.}

\section{Collection of 1885.}

Most of this collection was made in south eastern Oregon, during May, and June. I offer them at the very low price of ten dollers per set: 100 or more, your selection, six cents each : Less than 100 eight cents each.

\section{Yours respectfuly,}

THOMAs HoWELL, Arthur, Oregon U. S. A.

Money-order Office, Portland, Oregon.

756 Clematis Douglasii Hook.

757 Ranunculus cymbalaria Pursh 758 sceleratus L.

759 Delphinium decarum v. Nevad.

760 Pronia Brownii Dougl.

761 Canbya aurea Watson. n. sp.

762 Corydalis aurea v. occidentalis

763 Arabis eanescens Nutt.

$764 \quad$ Lyallii Watson.

765 Streptanthus Howellii Watson

766 Caulanthus pilosus Watson.

767 Vesicaria occidentalis Watson.

768 Draba alpina L. ?

769 Smelowskia Fremonti Watson

770 Erysimum cheiranthoides L.

771 Stanleya viridiflora Nutt.

772 Thelypodium Nuttallii Wats.

777 Viola Nuttallii, a smooth form.

778 Silene multicaulis Nutt. ?

779 Arenaria pungens Nutt.

781 Rhus toxicodendron $\mathrm{L}$.

782 Lupinus mierocarpus Lindl.

783 brevicaulis Watson.

784 ealearatus Kell.

785 argenteus Pursh-

786 holosericeus Nutt.

788 Trifolium Kingii Watson.

789 Plummeræ Lemmon.

730 Petalostemon ornatus Dougl

791 Astragalus Bourgovii Gray.

792 conjunetus Watson.

793 misellus Watson. n. sp.

794 Hypoglottis L.

795 diphysus Gray. var. eryptoides Gray.
800 Astragalus Mortoni Nutt.

801 diurnus Watson. n. sp.

802 Lathyrus palustris L.

803 polymorphus Nutt.

804 albus Watson.

805 Cercocarpus ledifolius Nutt.

806 intricatus Watson.

807 Geum triflorum Pursh.

808 Ivesia Baileyi Watson.

809 Peraphyllum ramosissimum.

810 Saxifraga reflexa Hook.

811 Heuchera rubescens Torr.

812 Ribes leptanthum Gray.

813

814

815 Gayophytum romosissimum.

816 Enothera cæspitosa Nutt.

817

818

819

820

$\times 21$

822

923

Nuttallii T. \& G. heterantha Nutt. trichocalyx Nutt. andina Nutt. Boothii Dougl. seapoidea Nutt.

825

826 Peucedanum leptocarpum Nut

827

828

829

830

8:1 Carum Oreganum Watson.

832 Caucalis microearpus H. \& A.

833 Valeriana sylvatiea Banks.

834 Dimeresia Howellii Gray. n. g.

835 Aplopappus lanuginosus Gray

$836 \quad$ Howellii Gray. n. sp.

837 Solidago serotina Ait.

838 Aster Fremonti Gray.

839

840

841

842

843 scopulorum Gray.

Engelmanni $v$.glaucescens. v. ledophyllus.

Erigeron Howellii Gray. compositus v. discoideus 
844 Erigeron peucephyllus Gray.

845

846 Bloomeri Gray.

847 Balsamorrhiza terebinthacea.

848 Wyethia helianthoides Nutt.

849 Hymenopappus filifolius $\mathrm{Hk}$.

850 Chænactis Nevii Gray.

851 Tanacetum potentilloides Gr.

852 Artemisia spinescens Eaton.

853 Tetradymia spinosa H. \& A.

854 Arnica alpina Olin. var.

855 Senecio canus Hook.

856 aureus v. borealis T. \& $\mathrm{G}$.

857 hydrophyllus Nutt.

858 Crepis runcinata T. \& G.

859 var. hispidulosa.

860 occidentalis Nutt. var.

861 Prenanthes alata Gray.

862 Troximon Nuttallii Gray.

863 glaueum v. lanceolatum.

864 alpestre Gray.

865 Gilia capillaris Kell.

866 pungens Benth.

867 minima Gray.

868 floceosa Gray.

869 congesta Hook,

870 tenerrima Gray.

871 Polemonium flavum Greene.

872 Nemophila breviflora Gray.

873 Phacelia Ivesiana Torr.

874 ciliata Benth.

875 bicolor Torr.

876 Emmenanthe parviflora Gray.

877 Conanthus aretioides Watson:

878 Hesperoehiron Californicus,

879 Heliotropium Curassavicum I.

880 Echinospermum diffusum.

881 Redowskii v. cupulatum.

882 Krynitzkia eireumscissa Grya.

883

884

885

886

887

888

oxycarya Gray.

affinis Gray.

Pattersoni Gray. pterocarya $\mathrm{v}$ pectinata.

Mertensia lanceolata DC. oblongifolia Don.

890 humilis Nutt.

891 var. ?.

892 deustus Dougl, var.

893 Gairdneri Hook.

894 ? n. sp.

895 Mimulus Bigelovii $\mathbf{G}$ ray.

896 var, ovatus Gray.

897 dentatus Nutt.

898 nasatus Greene.

899 pedunculatus Dougl.
900 Synthyris rubra Benth.

901 Aphyllon comosum Gray.

902 Boschniakia strobilacea Gray.

903 Scutellaria nana Gray.

904 Plantago pusilla Nutt.

905 Chorizanthe Watsonii T. \& G.

906 Eriogonum cæspitosum Nutt.

907 ovalifolium Nutt.

908 var. proliferum Wat.

909 Rumex — ?

910 Polygonum Douglasii Greenc.

911 Atriplex pusilla Watson.

912 confertifolia Watson.

913 Monolepis pusilla Torr.

914 Chenopodium leptophyllum.

915 Nitrophila occidentalis Wats.

916 Shepherdia Cunadensis Nutt.

917 Celtis retieulata Torr.

918 Parietaria Pennsylvanica Muhl

919 Callitriche autumnalis L.

920 Salix lasiolep;s v. Bige ovii Be.

921 Allium Nevii Watson.

922

923

924

925 Leucocrinum montanum Nutt.

926 Camassia Litehlinii Watson.

927 Fritillaria atropurpurea Nutt.

928 biflora Lindl.

929. Potamogeton rufeseens Sehrarl

930 Scirpus Olneyi Gray.

931 Nevadensis Watson.

9:2 sylvaticus v, digynus $\mathrm{B} 0$

933 Carex filifolia Nutt.

$934 \quad$ Geyeri Boott.

935

936

937

938

939 Spartina gracilis Trin.

940 Stipa oecidentalis Thurb.

941 Sporobolus depaup $v$. nedosus

942 Agrostis microphylla Steud.

943 Deyeuxia neglecta kith.

944 Melica fugax Bol.

945 strieta Bol.

946 Poa Galifornica Vasey.

947 var.

948 Nevadensis Vasey.

949 var. elongata Vasey.

950 var. glyceroides Vasey.

951 Glyceria Lemmoni Vasey.

952 Festuca confinis Vasey.

953 Bromus breviaristatus Thurb.

954 Agropyrum glaucum Vasey.

955 divergens v, tenue Vas. 
THOMAS HOWELL.

Arthur, Oregon.

956 Clematis ligusticifolia Nutt.

957 Anemone occidentalis Watson.

958

959 Trautvetteria grandis Nutt.

960 Ranuneulus hispidus var. Oregonus Gray.

961 oceidentalis var. Lyallii Gray

962 Myosurus apetalus v. lepturus G.

963 Delphinium simplex 10ougl.

964 pauciflorum Nutt. (D. sim

96- Nuphar plex of former distributions.

963 Cardamine pratense L.

937 pratense var. occidentalis.

9.38 Erysimum asperum DC.

969 Lepidium virginieum L.

970 Lyehnis Californicus Watson.

971 Calandrinia Columbiana Howell

972 Claytonia parvifolia Mocq.

973 Sidaleea

974 Mellilotus parviflora Desf.

975 Trifolium microcephalum Pursh

970 Psoralia lanceolata Pursh.

977 Lathyrus Nuttallii Watson.

978 ochroleucus Hook.

979 Spiræa betulæefolia v. rosea $\mathrm{Gr}$.

980 Eriogynia pectinata Hook.

931 Potentilla glandulosa Iíndl.

932 Saxifraga Tolmiei F. \& G.

983 Boykinia major Gray.

984 Bolandera Oregana Watson.

985 Heuchera cylindrica Dougl.

986 Seedum Douglasii Hook.

937 Hippuris vulgaris L.

988 Myriophyllum hippurioides Nut

98) Lythrum Hyssopifolia L.

990 Epilobium minuium Lindl.

991 Boisduvalia densiflora Watson.

992 Angelica genuflexa Nutt.

993 Angeliea-?

924 Ferula dessoluta Watson.

995 Viburnum elleptieum Hook.

996 Brickellia grandiflora Nutt.

997 Chrysopsis villosa Nutt.

998 Aster occidentalis Nutt.

992 Hallii Gray.

1007 Erigeron salsuginosus G:ay.

1001 Oregana Gray.

1002 Gnaphalium mier jeophalin

1003 Madia sativa Molin.

1001 Matricaria discoidea DC:

\05 Senecio Fremonti var. oceidentalis Gray.
1006 Stephanomeria minor Nutt.

1007 Crepis virens L.

1008 Troximon laciniatum Gray.

1009 Bryanthus empetriformis Gray.

1010 Chimaphila umbellata Nutt.

1011 Pyrola aphylla Smith.

1012 secunda $\mathrm{L}$.

Dodecatheon frigidum var. dentatum Gray.

1014 Appocynum androsamæfolium.

1015 Asclepias speciosa Torr.

1016 Hydrophyllum occidentale Gr.

1017 Nicotiana attenuata Torr.

1018 Verbena bracteosa Michx.

1019 Stachys palustris L.

1020 Chamissonis Benth.

021 Abronia millifera Dougl.

022 Oxyria digynia Campd.

1023 Urtica Lyallii Watson.

1024 Salix Hookeriana Barratt.

.025

1026

1027 cordata Muhl., a form. glauca v. villosa And.

Barclayi And.?

ysichiton Kamtschatense Sch.

1029 Listera convallarioides Nutt.

1050 Rrodiza grandiflora Smith.

031 Erythronium - ?

10.2 Calochortus macrocarpus Doug

1033 longibarbatus Watson.

t034 Trillium sessile var. Californicum Watson.

1035 Veratrum Californicum Watson

1036 Xerophyllum tenax Nutt.

1087 Luzula spadicea $v$. melanocarpa

1038 Carex

1039

1040

1041

$10 \leftarrow 2$

1043

1044

1045

1046

stis Howellii Seribner.

1047 Aira caryophylla L.

$10: 8$ Pleuropogon refractum Benth.

10.9 Poa oecidentalis $\mathrm{V}$. \& S.

10:0 (łlyceria distans Wahl.

1051 Festuca Howellii Hackel.

10.52

1053 occidentalis Hook? mierostachys var pauei-

Breweri kioott.

- nuhl.

Hendersonii Bailey.

.

eryptocarpa Meyer. acuta v. prolixa Bailey

flora Scribner.
1054 Bromus ciliatus v. pauciflorns.

1055 Elvmus Amerieanus V. \& S. 



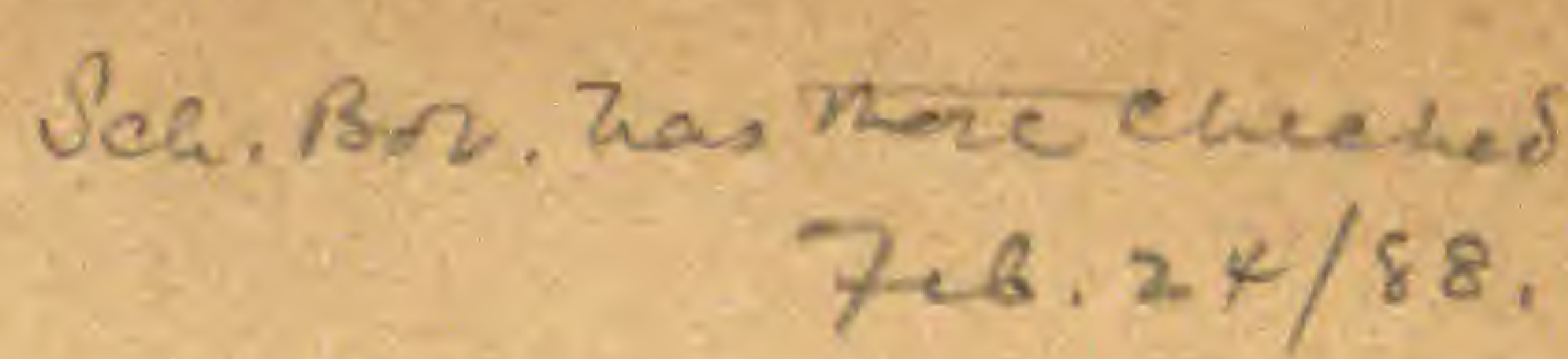

\section{HOWELI'S PACIFIC COAST PLANTS. \\ COLLECTION OF, 1887.}

Having spent most of the past summer in South Western Oregon, I am able to offer a very fine lot of new and rare species from that interesting region. The new species are set in Italies.

Piics; full sets, five dollars per century, desiderata six dollars per century. Less than 100, eight cents each.

\section{TH0MAS HOWELL,}

ARTHUR, OREGON.

1056, Anemone nemorosa $\mathrm{L}$.

1057, Ranunculus occidentalis var. Rattani Gray.

v1058, R. hebecarpus H. \& A.

$\checkmark 1059$, Cimicifuga elata Nutt.

$\checkmark 1060$, Darlingtonia Californica Torr.

$\checkmark$ 1061, Esthscholtzia tenuifolia Hook.

$\checkmark 1062$, Arabis I,yalli IVatson. ? Not the same as No.764 of iny distribution of 1885 , which is A. microphylla Nutt.

1063, Mrabis Howellii Watson

$\checkmark 1064$, A. purpurascens Howell.

1065 , A. subpimatifida Watson

$\checkmark$ 1066. Streptanthus tortuosus Kell.

1067, Cardimine paucisecta Benth.

1068. C. Breweri Watson.

-1069, Dentaria gemmata Watson.

$\checkmark 1070$, Draba brachycarpa Nutt.

$\checkmark 1071$, Lepidium nitidum Nutt.

$\checkmark 1072, L$. Oreganum. Distinguished from L. dictyotum, by its more erect habit, and smooth, not prominently reticulated, divergent-toothed pods.

1073 , Thlaspi alpestre L.

1074, Thysanocarpus radians Benth.

$\checkmark 1075$, Cleome lutea Hook.

1076, Viola palustris L.

1077, V. primulæfolia var. occidentalis Gray.

4078, V. præmorsa Dougl.

-1079 , V. chrysantha Hook.

1080 , V. Sheltoni Torr

1081. V. lobata Benth.

1082 , V. lobata var. integrifolia Watson.

1083 , V. cuneata Watson.

$1084, \mathcal{V}$. Howellii Gray. Distributed as V. canina var. sylvestris in 1884 .

1085, Silene campanulata Watson.

$\checkmark 1086, S$. longistylis Engelm.

1087, Cerastium arvense var. angustifolium Fenzl.

1088, Stellaria nitens Nutt.

$\checkmark 1089$, S. longipes Goldie.

-1090, s. longifolia Muhl. ?

$\checkmark$ 1091, Arenaria congesta Nutt.

1092, A. peploides var. oblongifolia Watson. 
1093. Calandrinia Nevadensis Gray.

1094, C. oppositifolia Watson.

v1095, C. Leana Porter.

1096, C. Cotyledon Watson.

1097, C. mierantha Schlecht.

1098, Claytonia asarifolia Bong.

v 1099, C. spathulata var. tenuifolia Gray.

$\sim 1100$, Sidalcea spicata Greene.

1101, S. Oregana Gray.

1102 , S. . campestris Greene ?.

1103, Erodium moschatum Willd.

1104, Limnanthes alba Hartweg. Not L. alba of former distributions, (which is a form of L. Douglesii or a new species,) but the true thing.

1105, Rhammus occidentalis. Shrub 5-10 feet high, with glabrous, corøaceous, oval to obovate, inch or two long, evergreen leaves: otherwise like R. Purshiana.

1106, Ceanothus cuneatus Nutt.

1107, Rhus aromatica var, triloba T.\& G.

- 1108, R. glabra L.

-1109, Lupinus Breweri Gray.

-1110, L. nanus Dougl.

$\checkmark 1111$, I. luteolus. Kell.

1112, Trifolium Breweri Watson.

vi13, T: Howellii Watson.

ᄂ 1114, T. Harneyensis. Stems erect, from creeping, perennial roots, 4-8 inches high ; leaf'ets linear to linear-lanceolate, irregularly dentate, 1-3 in. long; heads oblong, on rather long peduncles : flowers (even in bud) reflexed; calyx wooly. Harney Valley, S. E. Ogn.

$1115, \tau$. tridentatum var. ma Stout, 1-3 feet high, with large many-flowered liearde.

1116, T. tridentatum var. melananthum Watson.

1117, T. depauperatum Desv.

1118, Hosackia Torreyi Gray.

1119, Astragalus Hendersoni Watson. Fiowers omly.

- $1120, \not 1$ accidens Watson. Fruit only. The A. arr ectus of former distributions.

1121,21 sylvaticus.

-1122, Vicia gigantea Hook.

1123, Lathyrus polyphyllus Nutt.

v124, L. palustris L. var.

-1125, L. Torreyi Gray.

21126, Prunus subeordatus Berth.

1127, Rubus nivilis Dougl.

1128, Potentilla glandulosa var. Nevadensis Watson.

1129, Horkelia hirsuta Tindl.

$-1130, H$. latiloba. The $H$. congesta var. Iatiloba of 1884 .

$\sim 1131, H$. Hendersoni. Densly silky pubescent, not glandular, petals narrowly lanceolate, about as long as the calyx-lobes : otherivise like $H$. fusca. 
1132, Amplanchier alnifolia Nutt.

-1133, Saxifraga fragarioides Greene.

-1134 , S. Virginiensis Michx. var.

-1135, Chrysosplenium glechomæfolium Nutt.

1136, Tillæa minima Miers.

2 1137, Sedum stenopetalum Nutt.

1138, Cotyledon Oregonensis Watson.

1139, Epilobium glaucum. Perennial, 2-4 feet high, smooth and glaucous, or the inflorescens pubescent; leavs lanceolate, callus dentate, sessile; petals purple, half inch long or more, twice longer than the narrowlylanceolate calyx-lobes; stamens shorter than the barely excerted, style.

1140, Clarkia rhomboidea Dougl.

-1141, Gayophytum pumilum Watson.

-1142, Enothera strigulosa T. \& G.

1143, Godetia lepida var. Arnottii Watson.

1144, G. quadrivulnera Spach.

-1145 , Bois luvalia glabella Walp.

1146, Eryngium petфolatum Hook.

-1147, Deweya Kelloggii Gray.

$114 \diamond$, Carum Kelloggii Gary. ?

v1149, Sium cicutæfolium Gmelin.

-1150 , Glycosma occidentale Nutt.

1151, Phellopterus littoralis Schmidt.

1152, Ferula Californiea Gray.

1153, Peucedanum Howellii Watson.

-1154, $P$ microcarpum. Subcaulescent, 6-21 ins. high; leaves bipinnate, leaflets deeply cut into oblong-lanceolate segments; flowers yellow; carpels small.

1155, Cornus, Canadensis L.

1156, Garrya elliptica Dougl.

-1157, Symphoricarpos rotundifolius Gray.

-1158 , S. racemosus var. pauciflorus Gray.

1159, Lonicera conjugialis Kell.

-1160, L. hispidula Dougl.

1161, Galium bifolium Watson.

1162 , G. boreale L.

1163, G. Nuttallii Gray.

1164, G. Andrewsii Gray.

1165, Valerianella macrocera Gray.

-1166, V. aphanoptera Gray.

1167, Eupatorium occidentale Hook.

1168, Brickellia Greenei Gray.

1169, Chrysopsis Oregana Gray.

1170, Bigelovia Douglasii Gray.

1171, Erigeron Coulteri Gray. var.

$\checkmark 1172$, E. nudatus Gray.

$\checkmark 1173$, Stylocline filaginea Gray.

1174, Psilocarpus tenellus Nutt.

1175. Evax caulescens Gray. 
1176, Antennaria argentea Benth,

$\sim 1177, \mathrm{~A}$.

plantaginifolia Hook.

1178, Anaphalis margaritacea Benth. \& Hook.

1179, Gnaphalium palustre Nutt.

- 1180, Franseria Hookeriana Nutt.

1181, Rudbeckia Galifornica Gray.

-1182, Balsamorrhiza Hookeri Nutt.

1183, Helianthus annuus $\mathrm{L}$.

1184, Blepharipappus lavis Gray.

-1185, Madia elegans Don.

1186, M. sativa var. congesta T. \& G.

1187, Hemizonia luzulrefolia DC.

1188, H.

1189, H.

$1190, \mathrm{H}$.

Fitchii Gray.

1191, Lasthenia glaberrima DC.

1192, Bueria gracilis va. aristosa Gray.

-1193, Eriophyllum expitosum var, integrifolium Gray.

1194, Helenium Bigelovii Gray.

$\checkmark 1195$. H. autumnale $\mathrm{L}$.

1196, Cotula coronopifolia L.

1197, Artemisia Ludovieiana Nutt.

$\checkmark 1198$, A vulgaris var. Californica Bess.

1199, Luina hypoleuea Benth.

ᄂ1200, Arnica discoidea Benth.

\1201, Cnicus Breweri Gray.

1202, Microseris linearifolia Gray.

-1203, Hieracium eynoglossioides var. nudicaule Gray.

1204, Troximon aturantiacum Hook.

$1205, \mathrm{~T}$.

barbellulatum Greene.

$\checkmark 1206$, Lactuea pulchella DC.

ح 1207, Speeularia perfoliata A. DC. ? A slen ler form.

$\checkmark 1208$, Campanula prenanthoides Durrand.

1209 , Menziesia ferruginea Smith.

v 1210, Dodecatheon Hendersoni Gray.

-1211, D. Jeffreyi var. alpinum Gray.

- 1212, Androsace septentrionalis L.

-1213, Trientalis Europea var. arctica Ledeb.

1214, Fraximus Oregana Nutt.

-1215, Asclepias Mexicana Cav.

1216, Microcala quadrangularis Griseb.

1217, Menyanthes trifoliata L.

1218, Phlox adsurgens Torr.

41219, Gilia Harknessii Curran.

1220, G. lencocephala Gray.

1221, G. divaricata Torr.

1222, Hydrophyllum Virginicum L.

$\checkmark 1223$, Nemophila pedunculata Doug!.

-1224, Phacelia Rattani Gray.

1225, P. Pringlei Gray.

1226, Echinospermum Greenei Gray. 
- 1227 , Krynitzkia Chorisiana Gray.

- 1228, K. Torreyana Gray.

1229 , Plagiobothrys rufescens F. \& M.

*1230, Amsinckia lycopsoides Lehm.

1231, Mertensia paniculata Don.

1232, Solanum nigrum var. villosum Mill. ?

1233, Antirrhinum leptalizum Gray.

1234, Collinsia Torreyi Gray.

$\checkmark 1235$, Serophularia Californica Cham.

1236, Pentstemon Rattani Gray.

1237, P. Rattani var. minor Gray.

1238, P. Rattani var. Kleei Gray.

-1239, P.. Røzli Reg.

ᄂ240, P. heterophyllus Lindl.

-1241, P. azureus Benth.

1242, Minulus Douglasii Gray.

$\sqrt{ } 1243$, M- $\quad$ primuloides Benth. Small, wooly form.

4244 , M. luteus I.

1245, M. nisutus Greene. True; not the same as No.

898 of iny listribution of 1885 , which is something else.

$\checkmark 1246$, Orthocarpus pachystachyus Gray.

$\checkmark 1247,0$. lithospermoides Benth.

1248, Cordylanthus pilosus var. Bolanderi Gray.

- 1249, Verbena prostrata R. Br.

1250, Monardella villosa Benth.

$\checkmark 1251$, M. villosa var. glabella Gray.

-1252 , Scutellaria tuberosa Benth.

7253, S. angustifolia var. eanescens Gray.

1254, P’olygonum Douglasii var. latifolium Greene.

1255, P. Californieum Miesner.

$\sqrt{1256}$, Eriogonum datum Dougl.

1257, E. virgatum Benth.

1258, Urtica holosericea Nutt.

.1259, Euphorbia serpyllifolia Pers.

1260, Myrica Gale I.

1261, Salix macrocarpa Nutt.

1262, Quercus Kelloggii Newberry.

$1263, Q$. Sadleriana $R$. B . The Q.--? of my collection of 1884 .

1264, Phoradendron flavescens var. villosum Engelm.

$\checkmark 1265, \mathrm{P}$. juniperinum var. Libocedri Engelm.

1266, Chamæeyparis Lawsoniana Parl.

1267, Habenaria elegans Bol.

1268, Cephalanthera Oregana Riechenb. f.

1269, Cypripedium fasciculatum Kell.

$\checkmark .1270$, Mris bracteata Watson.

1271, Allium validum Watson.

1272 , A. tribracteatum Torr.

1273 , Brodiæa eapitata Benth. 
2.1275, Hastingsia alba Watson.

1276, Maianthemum bifolium var. dilatatum Wood.

1277, Lilium pardalinum var. angustifolium Kell.

1278, Fritillaria recurva Benth.

1279, Erythronium Hendersoni Watson.

$-1280, E$.

71281, E.

Howellii Watson.

1282, Calochortus citrinum Watson.

cholliz Watson.

1084, Prosartes trachyandra Torr.

1284, Trillium rivale Watson.

1285, Tofieldia occidentalis Watson.

1286, Narthecium Cølifornicum Baker.

1287, Sagittaria variabilis Engelm.

1288, Luzula comosa var. micrantha Watson.

1289, Juncus Bolanderi Engelm.

1290, J. patens E. Meyer.

c 1291, J. triformis Engelm.

1292 , J. longistylis Torr.

$\sqrt{1293}$, J. xiphoides var. triandrus Engelm.

$\sqrt{1294}$, J. oxymeris Engelm.

1295, Cyperus aristatus Rottb.

1296, Scirpus lacustris var. occidentalis Watson.

1297, S. maritimus L.

1298, S. lineatus Michx.

1299, Eriophorum russeolum Fries.

1300, Carex decidua Boott.

1301, C. laciniata Boott.

1302, C. Iuzulæefolia W. Boott.

1303 , C. multicaulis Bailey.

1304, C. polytrichoides Muhl.

c 1305, C. teretiuscula var. ramosa Boott.

21306, C. canescens var alpicola Wahl.

1307, C. straminea var, mixta. Bailey.

1308, Sporobolus depauperatus Vasey.

1309, Aristida oligantha Michx.

1310, Agrostis asperfolia Trin.

$1311, A$. exaratavar. angusta Vasey.

1312, 2 . microphylla var. major Vasey.

1313,2 . Microphylla var. laxa Vasey,

- 1314, .1. attenuata, Vasey.

1315, भ. Hallii Vasey.

-1316, Gastridium australe Beauv.

1317, Meliea aeuminata Bol.

1318, $M$. Harfordii var. minor Vasey.

- 1319, Poa tenuifolia var. stenophylla Vasey.

$1320, P$. argentea

1321, Glyceria

1322 , Festuca rubra L. var.

1323, Bromus Suksdorfia Vasey.

-1324, B.

$1325, \mathrm{~B}$.

Oreuttianus Vasey.

B. virens Nutt. ? 
1326, Hordium

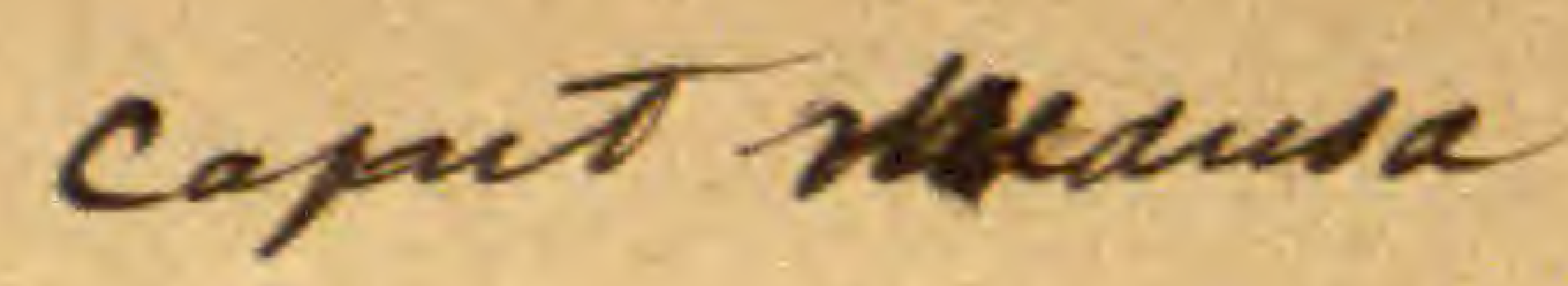

-1327, Elymus arenarius L.

1328, Equisetum lævigatum Al. Braun.

ᄂ 1329, Pellæa brachyptera Baker.

V 1330, Adiantum emarginatum Hook.

15. I make the following changes in name, of former sets, viz.

No. 7 , Ranunculus Flammula, is R. Flammula var. intermedius Hook.

No. I0, R.

No. $11, \mathrm{R}$.

Hookeri, is R. triternatus Gray.

No. 21, Delphinium simplex, is D. paueiflorum Nutt.

No. 62, Silene Lyallii, is S. Douglasii Hook.

No. 83, Claytonia exigna, is C. spathulata Dougl.

No. 101, Limnanthes alba, is L. Douglasii R. Br. or a new species,

No. $15 \mathrm{v}$, Astragalus arrectus, is A. accidens Watson.

No. 516, Urtica dioica, is U. gracilis Ait.

No. 602, Carex Houghtoni, is C Oregonensis Bailey.

No. 610, C. laxiffora var. plantaginea, is C. Hendersoni Bailey.

No. 630, Agrostis Scouleri, is A. Howellii Scribner.

No. $631, \mathrm{~A}$ varians, is A. Oregana Vasey.

No. 656, Poa Douglasii, is P.—? n. sp.

No. 660, P. stenantha, is perhaps P. Columbiana Dougl.

No. 762 , Corydalis aurea var. occidentalis, is C. curvisiliqua Engelm.

No. 761 , Arabis Lyallii, is A. microphylla Nutt.

No. 784, Lupinus calcaratus, is L. sulphureus Dougl.

No. 909, Rumex-? is R. salicifolius Wein.

No. 935, Carex —? is C. acuta L.

No. 936, C ? is C. Jamesii Torr.

No. 937, C. - ? is C. marcida var. debilis Bailey.

No. 938 , C. ? is C. festiva Dew.

No. 970, Lychnis Califormicus is Silene multicaulis Nutt.

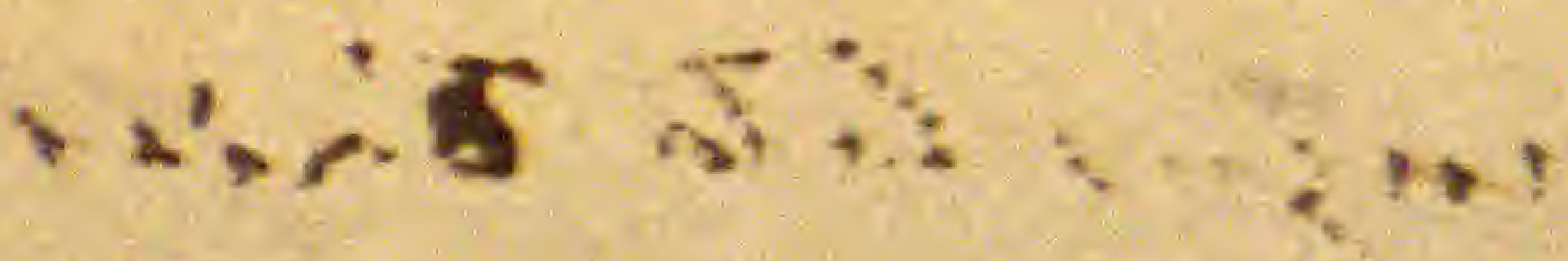





\section{HOWELL'S PACIFIC COAST PLANTS. COLLECTIGNS OF 1888 TO 1894 INCLUSIVE}

Not having flone much collecting sinee 1877 , I have not made any distribution since, that date. But having accumulated a few rare species I now offer the following collection at iny usual price; five dollars per 100 in full sets. Less than a full set, 8 cts. each.

Some of these specimens have been on hand for several years and are more or less faded, otherwise they ar in good order.

THOMAS HOWELL,

ARTHUR, OREGON.

1331 Ranuneulus ranus, Benth.?

1401 R. Californicus, Benth.. ?

$1332 \mathrm{R}$ maximus, Greene.

1485 Delphinitum ochroleuca, Nutt.

140.5 Berberis pumila, Greene. Eryth. I. 149.

1486 Lepidism divaricatum. Howell.

1487 Hrabis secunda, Howeli.

1408 Cardamine pulcherirma Greene. Eryth. I, 110.

$1488 \mathrm{C}$ Z Zobata, fowell.

1409 C. sinuata, Greene. Eryth. I, 110.

1410 Cheiranthus asper, Chain. \& Schlecht.

1333 Nusturtium palustre, DC.

$1334 \mathrm{~N}$. lyratum, Nutt.

1335 Draba lutea, Gilib.

1336 Heterodraba unilateralis, Greene.

1489 Viola Lunusdorffii, Fiseh.?

1490 Silere montana; Watson.?

1337 Stellaria Jamesii, Torr.

1338 Arenaria Howellii, Watson.

1491 Spraguea multiceps, Howell. The S. umbellata of my

1339 S. umbellata, Torr.

I492 Sidalcen campestris, Greene. Typical.

1493 Linum Lewisii, Pursh.

1340 Erodium macrophyllum, Hook. \& Arn.

1494 Oxalis Suksdorfii, Treleuse

1417 Ceanothus pumilus, Greene. Eryth. I, 149.

1495 Thermopsis gracilis Howell. Eryth. I, 109.

1341 Lupinus laxiflorus, Dougl.

$1494 \mathrm{~L}$. laxiforus var. mentamus, Howel.

1420 L. propinquus, Greene. Eryth. 1, 126.

1342 L. lepidns, Dougl.

1343 L. Drakei Hor'ell.

1344 L. holosericeus, Nutt.

1421 Trifolium barbigerum, Torr.

1345 T. Macrai var. dichotomum, Brewer.

$1346 \mathrm{~T} \quad$ Oregonum, Howell. Eryth I, 110. 
1347 Lotus Wrangelianus, F. \& M.

1350 Astragalus nigrescens, Nutt.

1351 A. Callifornicus, Greene.

1352 Vieia exicua, Nutt.

1355. Rubus lasiococcus, Gray.

1356 Horkelia tridentata, Torr.

1 196 Poterium annuum, Nutt.

1497 - Sorbus occidentalis, Greene.

1357 Amalanchier pallida, Greene.

1. 58 Saxifraga Marshallii, Greene

1359 S. ranuneulifolia, Hook.

1428 S. Mertensiana, Bong. Nonproliferous form.

1.498 s. Oregona, Howell.

1499 Mitella Hallii, Howell.

1500 Heuchera glabra, Willd.

1360 Parnassia Californica, Greene.

1361 Ribes velutinum, Greene.

1432 R. Menziesii, Pursh.

$14: 33$ R. amictum, Greene.

1.501 R. acerifolium. Howell

.502 Epilobium Hammondii, Howell

1362 E. rigidum, Hausskn.

1363 Enothera graciliflora, Hook. \& Arn.

1503 G. Hillgardii, Greene.

1504 E. ovata, Nutt.

1364 Sanicula bipinnata, Hook. \& Arn.

1365 S. Howellii, C \& R.

1366 Velæa Howellii, C. \& R.

1367 Peucedanum Canbyii, C. \& R.

1368 P. triturnatum var. brevifolium, C. \& R

1505 P. lævigatum, Nutt.

1506 Garrya buxifolia, Gray.

1370 Galium Bolanderi, Gray.

1371 Solidago Californica, Nutt.

1372 Sericocarpus Oreganus, Nutt.

1373 Aster briekelloides, Greene.

1507 Erigeron Siskiyouensis, Howell.

1508 E. speciosus, DC.

1509 E. Philadelphicus, L.

1441 Helianthus exilis, Gray.

1374 Madia elegans, Don.

1510 Cacaliopsis nardosmia, Gray.

1375 Senecio vestitus, Howe?l.

1511 S. hesperius, Greene.

1376 Microseris Douglasii, Gray.

1377 Scorzonella pratensis, Greene.

1378 Arctostaphylus manzanita, Parry.

1512 A. viscida, Parry.

1379 Arbutus Menziesii, Pursh. 
1513 Lysimachia thyrsiflora, L.

1380 Phlox longifolia var. Stansburyi, Gray.

1514 Gilia achilleæfolia, Benth.

1381 Polemonium carneum, Gray.

1515 Phacelia nemoralis, Greene.

1516 P. verna, Howell.

1282 Plagiobothrys Shastensis, Greene.

1386 Cryptantha rostellata, Greene.

1517 Pentstemon glandulosus, Lindl.

1518 P. Gairdneri, Hook.

1519 P. ovatus, Dougl.

1454 Diplacus glutinosus, Nutt.

1469 Orthocarpus erianthus, Benth.

1387 Pedicularis densiflora, Benth.

1389 Mirabilis Greenei, Watson.

1390 Eriogonum pendulum, Watson.

1391 E. thymoides, Benth.

1520 E. $\quad$ sphærocephalum, Dougl.

1392 Euphorbia glyptosperma, Engelm.

1473 Empetrum nigrum, L.

1521 Betula glandulosa, Michx.

1393 Salix lævigata var. angustifolia, Bebb.

1475 Iris Douglasiana, Herb.

1394 Allium Bolanderi, Watson.

1395 Camassia Howellii, Watson.

1396 Calochortus uniflorus, Hook. \& Arn.

1522 Erythronium montanum, Watson.

1523 Tolfieldia occidentalis, Watson.

1478 Luzula comosa, Meyer. var.

1397 Sparganium simplex, Hudson.

1524 Alopecurus Howellii, Vasey.

1398 Scribneria Bolanderi, Hack.

1399 Aspidium Nevadense, Eaton.

1525 Isoetes nuda, Engelm.

1526 I. Nuttallii, A. Braun. 



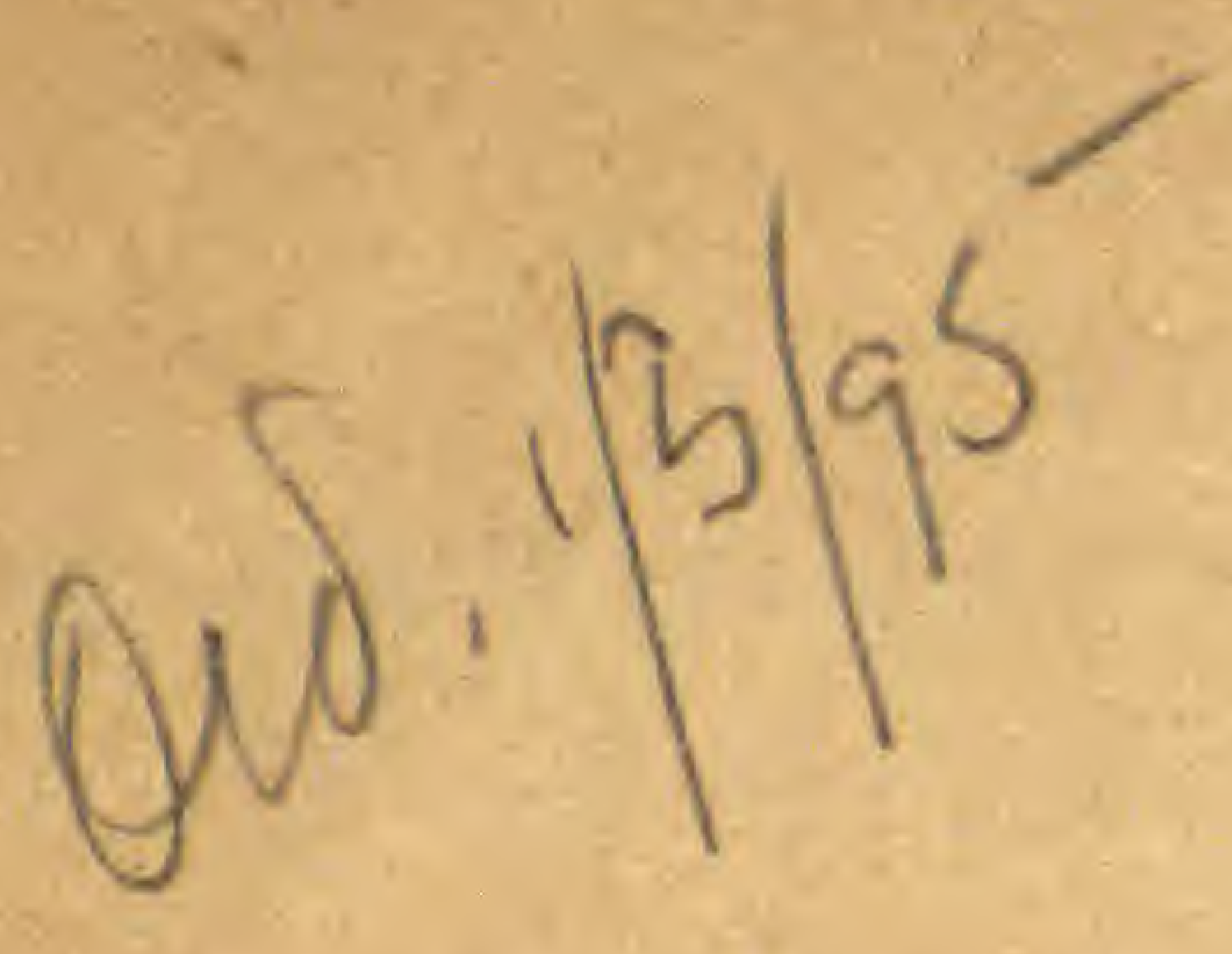

QKinn Db

\section{HOWELL'S PACIFIC COAST PLANTS. COLLECTIGNS OF 1888 TO 1894 INCLUSIVE} H64 fase. 5

Not having done mueh collecting since 1877 , I have not made any distribution since that date. But having accumulated a few rare species I now offer the following collection at iny usual price; five dollars per 100 in full sets. Less than a full set, 8 cts. each.

Some of these specimens have been on hand for several years and are more or less faded, otherwise they ar' in good order.

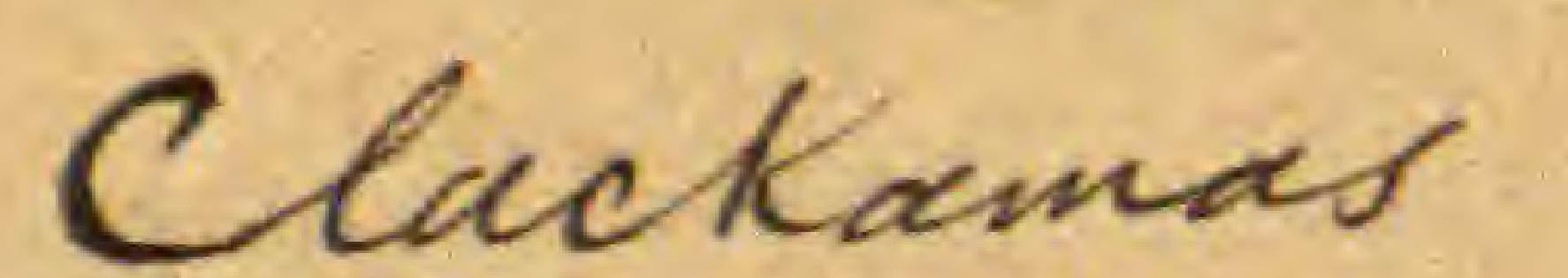

THOMAS HOWELL,

-1331 Ranunculus manus, Benth.?

1401 li. Californicus, Benth.. ?

$1332 \mathrm{k}$ inaximus, Greene.

-1485 Delphinium ochroleuca, Nutt.

-1405 Berberis pumila, Greene. Eryt

- 1486 Ber. 149.

- Lepidium darariculam, Howell.

- 1487 Mrabis secunda, Howell.

1408 Cardanine pulcherirma Greene. Eryth. I, 148

$7488 \mathrm{C}$. labata, fiowell.

1409 C. sinuata, Greene. Eryth. I, 148

1710 Cheiranthus asper, Cham. \& Sehlecht.

C.2

1033 Nasturtiun palustre, DC.

1334 N. lyratum, Nutt.

1335 Draba lutea, Gilib.

-1336 Heterodraba unilateralis, Greene.

1489 Viola Lin rsiorffii, Fisch.?

1490 Silene montana, Watson.?

1337 Stellaria Jamesii, Torr.

- 1338 Arenaria Howellii, Watson.

1491 Spraguea multiceps, Howell. The S. umbellata of my

- 1339 \&. umbellata, Forr.

- I492 Sidalcea campesiris, Greene. Typical.

1493 Linum Lesvisii, Pursh.

1340 Erodium macrophyllum, Hook \& Arn.

1494 Oxalis Suksdorfii, Trelease

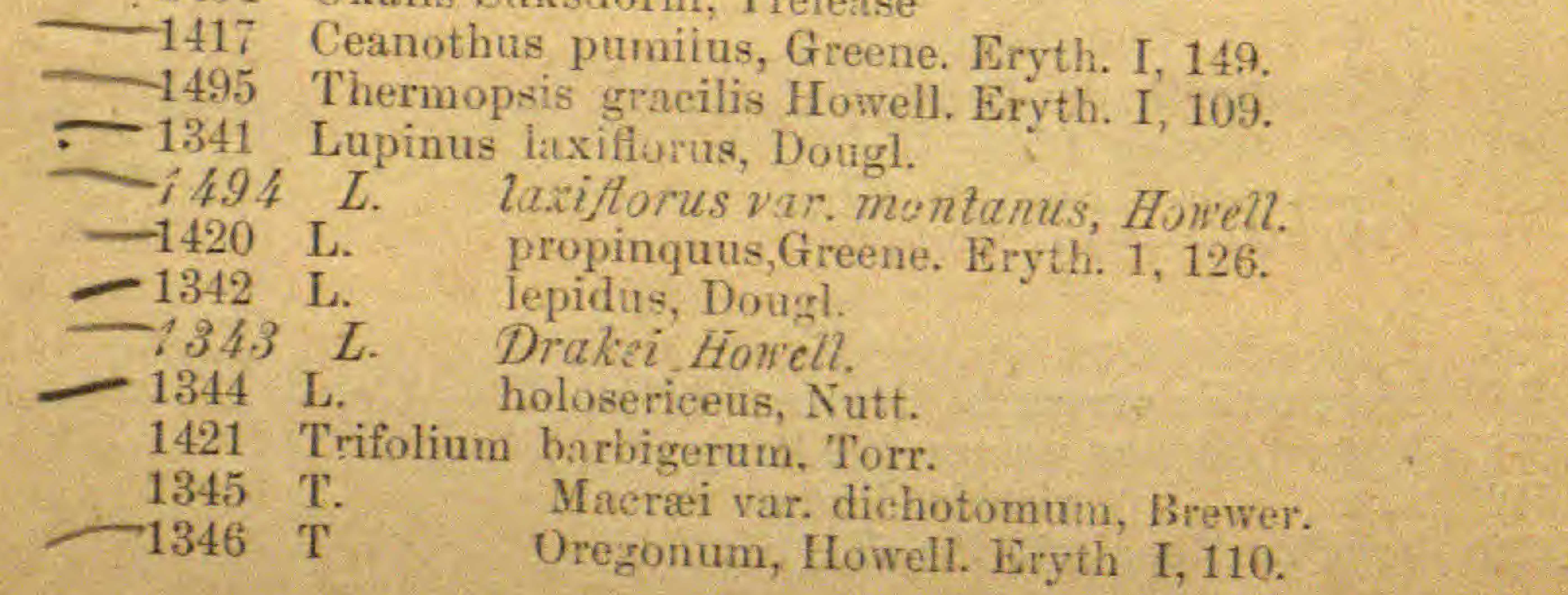

Mo. Bot. Garden,

1895. 


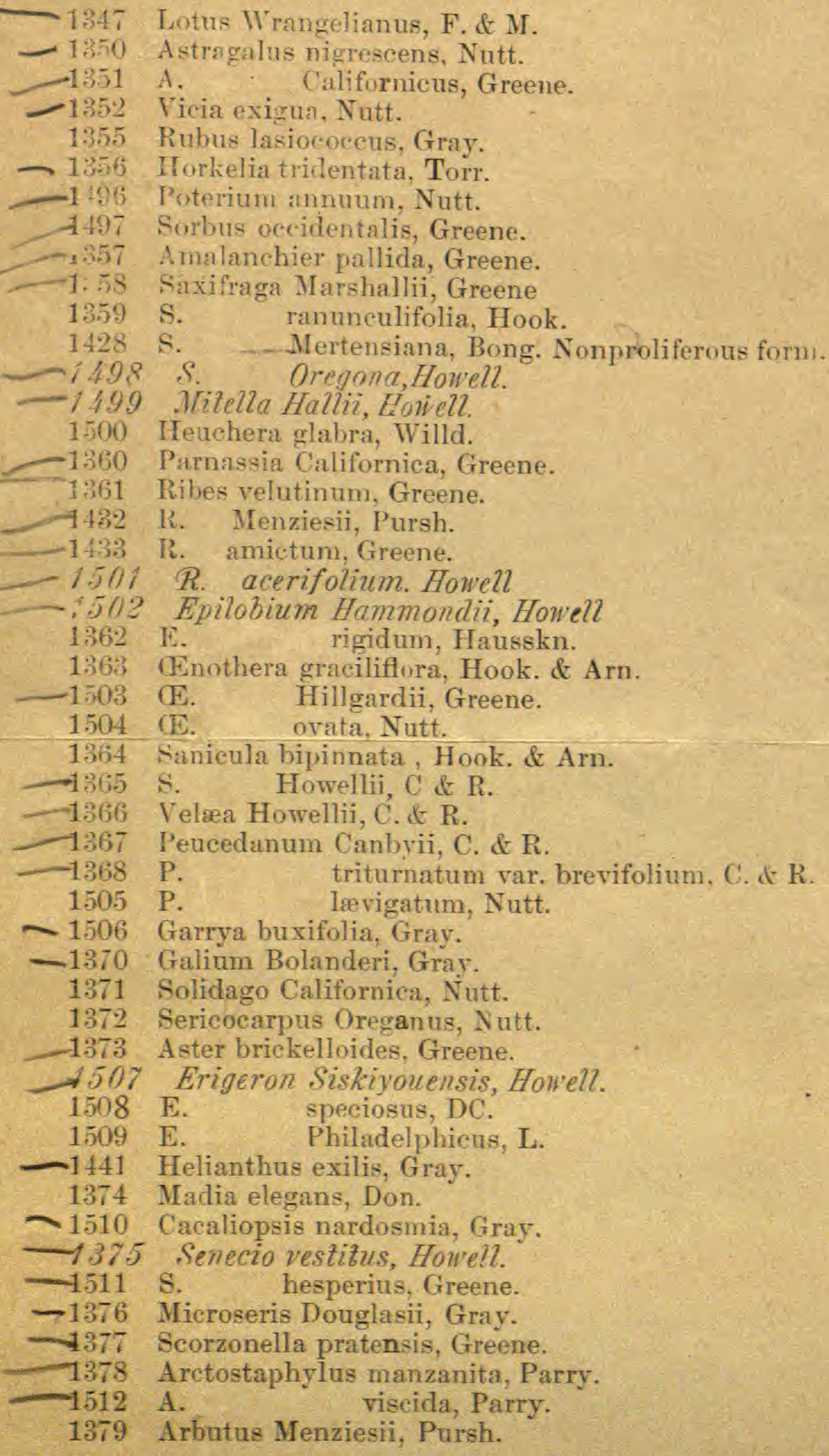


1513 Lysimachia thyrsiflora, L.

1380 Phlox longifolia var. Stansburyi, Gray.

1514 Gilia achilleæfolia, Benth.

-1381 Polemonium carneum, Gray.

-1515 Phacelia nemoralis, Greene.

I 16 P. verna, Howell.

1,82 Plagiobothrys Shastensis, Greene.

1386

Cryptantha rostellata, Greene.

1517 Pentstemon glandulosus, Lindl.

$\rightarrow 1518 \mathrm{P}$

P. Gairdneri, Hook.

P: ovatus, Dougl.

1454 Diplacus glutinosus, Nutt.

-1469 Orthocarpus erianthus, Benth.

1387 Pedicularis densiflora, Benth.

1389 Mirabilis Greenei, Watson.

1390

1391

Eriogonum pendulum, Watson.

E. thymoides, Benth.

E. sphærocephalum, Dougl.

1392 Euphorbia glyptosperma, Engelm.

-1473 Einpetrum nigrum, L.

1521 Betula glandulosa, Michx.

1393 Salix lævigata var. angustifolia, Bebb.

1475 Iris Douglasiana, Herb.

-1394 Allium Bolanderi, Watson.

$-1395$

Camassia Howellii, Watson.

1396 Calochortus uniflorus, Hook. \& Arn.

1522 Erythronium montanum, Watson.

1523 Toffieldia occidentalis, Watson.

$\longrightarrow 148$ Luzula comosa, Meyer. var.

1397 Sparganium simplex, Hudson.

1524 Alopecurus Howellii, Vasey.

1398 Scribneria Bolanderi, Hack.

1399 Aspidium Nevadense, Eaton.

1525 Isoetes nuda, Engelm.

1526 I. Nuttallii, A. Braun. 



\section{HOWELL'S PACIFIC COAST PLANTS.}

\section{COLLECTIGNS OF 1888 TO 1894 INCLUSIVE}

Not having done much collecting since 1877, I have not made any distribution since that date. But having accumulated a few rare species I now offer the following collection at iny usual price; five dollars per 100 in full sets. Less than a full set, 8 cts. each,

Some of these spccimens have been on hand for several years and are more or less faded, otherwise they are in good order.

THOMAS HOWELL,

ARTHUR, OREGON.

1391 Ranunculus uanus, Benth.?

1401 R. Califormicus, Benth.. ?

1332 R maximus, Greene.

1485 Delplinim ochroleuca, Nutt.

1405 Berberis pamila, Greene. Eryth. I. 149.

1486 Lepidium divaricalum. Howell.

1487 Nrabis secunda, Howell.

1408 Cardamine pulcherirma Greene. Eryth. I, 110.

$1488 \mathrm{C}$. lobata, fiowell.

1409 C. sinuata, Greene. Eryth. I, 110.

$1 \frac{110}{}$ Cheiranthus asper, Cham. \& Schlecht.

1333 Nasturtium palustre, DC.

1334 N. Iyratum, Nutt.

1335 Draba lutea, Gilib.

1336 . Heterodraba unilateralis, Greene.

1489 Viola Langsdorffi, Fisch.?

1490 Silene isontana, Watson,?

1337 Stellaria Jamesii, Torr.

1338 Arenaria Mowellii, Watson.

1491 Spraguea multiceps, Howell, The S. umbellata of $\mathrm{my}$

1339 s. former distributions; not of Torrey.

I492 Sidalem umbellata, Torr.

1493 Linum Lewisii, Pursh.

1340 Erodium macrophyllum, Hook. \& Arn.

1494 Oxalis Suksdorfii, Trelease

1417. Ceanothus pumilas, Greene. Wryth. I, 149.

1495 Thermopsis gracilis Howell. Eryth. 1, 109.

1341 Lupinus laxifloras, Dougl.

1494 L. Zuxiflamsvar. muntanus, Howell.

1420 L. propinquas, Greene. Bryth. 1, 128.

1342 L. Jepidus, Dougl.

i343 L. Drakei Howell.

1344 L. holosericeus, Nutt.

1421 Trifolium harbigenum. Torr.

$1345 \mathrm{~T}$. Macrei var, dichotomum, Brewer.

$1346 \mathrm{~T} \quad$ Oregonum, Howell. Eryth I, 110.

Mo. Bot, Garden. 
1847 Lotus Wrangelianus, F. \& M.

13,50 Astragalus nigrescens, Nutt.

1351 A. Californicus, Greene.

1352 Vicia exigun, Nutt.

1355 Rubus lasiococeus, Gray.

1356 Horkelia tridentata, Torr.

1490 Poterium annuum, Nutt.

1497 Sorbus occidentalis, Greene.

1957 Amalanchier pallida, Greene.

1. 58 Faxifraga Marshallii, Greene

13.59 S. ranunculifolia, Ḣook.

1428 S. Mertensiana, Bong. Nonproliferous form.

1498 S. Oregona, Howell.

1360 Parnassia Californica, Greene.

1361 Ribes velutinum, Greene.

1432 R. Menziesii, Pursh.

1433 R. amictum, Greene.

$1501 R$ acerifolium. Howell

.502 Epilobium Hammondii, Howell

1362 E. rigidum, Hausskn.

1363 Enothera graciliflora, Hook. \& Arn.

1503 (E) Hillgardii, Greene.

1504 G. - ovata, Nutt.

1364 Sanicula bipinnata, Hook. \& Arn.

1365 S. Howellii, C \&. R.

1366 Velæa Howellii, C. \& R.

1367 Peucedanum Canbyii, C. \& R.

1368 P. triturnatum var, brevifolium. C. \& R. .

1505 P. lævigatum, Nutt.

1506 Garrya buxifolia, Gray.

1370 Galium Bolanderi, Gray..

1371 Solidago Californica, Nutt.

1372 Sericocarpus Oreganus, Nutt.

1373 Aster brickelloides, Greene.

1507 Erigeron Siskiyouensis, Howell.

1508 E. speciosus, DC.

1509 E. Philadelphicus, L.

1441 Helianthus exilis, Gray.

1374 Madia elegans, Don.

1510 Cacaliopsis nardosmia, Gray,

1375 Senecio vestitus, Howe?l.

$1511 \mathrm{~S}$. hesperius, Greene.

1376 Microseris Douglasii, Gray.

1377 Scorzonella pratensis, Greene.

1378 A retostaphylus manzanita, Parry.

1512 A. viscida, Parry.

1379 Arbutus Menziesii, Pursh. 
1513 Lysimachia thyrsiflora, L.

1380 Phlox longifolia var. Stansburyi, Gray.

1514 Gilia achilleæfolia, Benth.

1381 Polemonium carneum, Gray.

1515 Phacelia nemoralis, Greene.

1516 P. verna, Howell.

1;82 Plagiobothrys Shastensis, Greene.

1386 Cryptantha rostellata, Greene.

1517 Pentstemon glandulosus, Lindl.

1518 P.

Gairdneri, Hook.

15. P. ovatus, Dougl.

1454 Diplacus glutinosus, Nutt.

1469 Orthocarpus erianthus, Benth.

1387 Pedicularis densiflora, Benth.

1389 Mirabilis Greenei, Watson.

1390 Eriogonum pendulum, Watson.

1391 E. thymoides, Benth.

1520 E. sphæroeephalum, Dougl.

1002 Euphorbia glyptosperma, Engelm.

1473 Empetrum nigrum, L.

1521 Betula glandulosa, Michx.

1393 Salix lavigata var. angustifolia, Bebb.

1475 Iris Douglasiana, Herb.

1394 Allium Bolanderi, Watson.

1395 Camassia Howellii, Watson.

1396 Calochortus uniflorus, Hook. \& Arn.

1522 Erythronium montanum, Watsoh.

Tर20 Toffieldia occidentalis, Watson.

1478 Luzula conrosa, Meyer. var.

1397 Sparganium simplex, Hudson.

1524 Alopecurus Howellii, Vasey.

1398 Seribneria Bolanderi, Hack.

1399 Aspidium Nevadense, Eaton.

1525 Isoetes nuda, Engelm.

1526 I. Nuttallii, A. Braun. 

243 Gaura parviflora Dougl.

248 Sanicula Nevadensis Natson.

249 S. brpisuatifida Dougl.

251 Osmorrhiza nuda T'orr.

257 Peucedauum leiocarpum Nutt.

$261 \mathrm{P}$

$262 \mathrm{P}$.

$263 \mathrm{P}$.

$264 \mathrm{P}$

266 Fatsia horrida B.\& $\mathrm{H}$.

utrieulatum Nutt.

Grayi C. \& R.

macrocarpum Nutt.

ferinosum Geyer

267 Cornus Nuttallii Audubon.

268 C. pnbescens iv utt.

271 Sambucus racemosa var. arborescens

272 Linnaea borealis var. longiflora Torr.

273 Symphoricarpos racemosus Michx.

275 Lonicera involucrata Banks.

278 Galium A parine var. Vaillantii Koch.

$280 \mathrm{G}$. trifidum $\mathrm{L}$.

282 Valerianella congesta Lincil

$283 \mathrm{~V}$. anomala Gray

286 Grindelia nana $N$ itt.

287 G. diseoidea Nutt.

289 Aplopappus Bloomeri Gray.

293 Solidago elonguta Nutt.

$295 \mathrm{~S}$. humilis var. nana Gray.

296 Sericocarpus rigidus Lindl.

300 Aster Đou glasii Lindl.

302 A. pulchellus Eaton.

306 Erigeron poliospermus Gray.

309 E. filifolius Nutt.

310 E. decumbens Nutt

311 E. inornatus Gray.

314 Micropus Californieus F. \& M.

315 Psilocarpus Oreganns Nutt.

316 Antennaria dimorpha T. \& G,

322 A. dioica Gairtn.

327 Balsamorrhiza sagittata Nutt.

331 Madia Nuttallii Gray.

333 M. filipes Gray.

334 Hemizonella Durandi Gray.

336 Layia glandulosa H. \& A.

339 Rigiopappus leptociadus Gray.

340 Chaenactis Douglasii H. \& A.

342 Crocidium multicanle Hooker,

346 Senecio fastigiatus Nutt.

348 Amica cordifolia Hooker.

354 Mieroseris nutans Gray.

$355 \mathrm{M}$.

troximoides Gray.

363 Troximon heterophyllnm Greene..

364 Downingia elegans Torr.

365 Howellia aqnatalis Gray.

366 Githopsis specularioides Nntt,

369 Heterocodon rariflorum Nutt.

370 Vaccirium oecidentale Gray.

$374 \mathrm{~V}$.

$375 \mathrm{~V}$. parvifolinm Smith.

ovaturn Pursh.

376 Arctostaphylos tomentosa Dougl.

378 Kalmia glauca Ait.

379 Menziesia glabella Gray.

380 Rbudodendron albiflorum Hooker.

$381 \mathrm{R}$. occidentale Gray.

386. Sarcodes sanguinea Torr.

388 Trientalis latifolia Hooker.
389 Glaux maritima L.

391 Erythraea Douglasii Gray.

394 Phlox Douglasii Hooker.

396 P. longifolia Nutt.

317 P. speciosa Pursh.

398 Collomia grandiflora Dougl.

$400 \mathrm{C}$ gracilis Dougl

401 Navarretia heterophylla Benth.

404 Gilia Bolanderi Gray.

405 G. tenella Benth.

406 G. intertexta Steud.

407 G. aggregata Spreng.

408 G. debilis Watson.

t09 G. inconspicua Dougl.

410 Polemonium humile Willd.

412 P misanthum Benth.

413 Hydroplyllum capitatum Dougl.

414 Nemophila parviflora Dougl.

415 N. Menziesii H. \& A.

418 Phacelia Menziesii Torr.

419 Romanzoffia Sitchensis Bong.

420 Hesperochiron pumilis Porter.

423 Cynoglossum grande Dougl.

424 Echinospermum Redowskii var.

426 Krynitzia Californica Gray.

$427 \mathrm{~K}$. Scouleri Gray.

428 Plagiobothrys nothofulvus Gray.

$431 \mathrm{P} \quad$ glomeratus Gray.

433 Amsinckia lycopsoides

434 Lithospermum pilosum Nutt.

431; Convolvulus Californicus Chois.

438 Collinsia pusilla

439 C. sparsiflora F. \& M.

440 C. parviflora Dougl.

442 C. linearis Gray.

443 Tonella collinvioides Nutt.

448 Pentstemon procerus Dougl.

451 Mimulus nanus H. \& A.

$453 \mathrm{M} . \quad$ cardinalis Dougl.

$454 \mathrm{M}$. alsinoides Benth.

459 M. pilosus Watson.

459 Gratiola ebracteata Benth.

460 Limosella aquatica $L$.

461 Synthyris rotundifolia Gray.

$462 \mathrm{~S}$. reniformis Benth.

466 Castilleia miniata Dougl.

468 Orthccarpus pilosus Watson.

$469 \mathrm{O}$.

$470 \mathrm{O}$.

4710.

4720. attenuatus Gray. castilleoides Benth. bracteosa Benth. pusillus Benth.

478 Aphyllon fascieulatum Gray.

479 Trichostema oblongum Benth.

$480 \mathrm{~T}$. lanceolatum Benth.

481 Monardella odoratissima Benth.

483 Micromeria Douglasii Benth.

485 Lophanthus urticifolins Benth

491 Plantago Patagonica Jacq.

497 Polygonum mininum Watson.

$498 \mathrm{P}$.

$500 \mathrm{P}$.

$503 \mathrm{P}$. coarctatum Dougl. imbricatum Nutt. boreale Small. Distributed as P. Davisiae Brewer. 
506 Eriogonum sphaerocephaluin Dougl.

507 E. umbellatum Torr

515 Umbellularia Californica Nuti.

517 Eremocarpus setigerus Benth.

519 Betnla oceidentalis Hook

523 Salix longifolia Muhl.

$529 \mathrm{~S}$. amygdaloides Arders.

$534 J$ aniperus oecidentalis Fook.

535 Thuya gigantea Nutt.

588 Corallorhiza Mertensiana Bong.

$5 \%, \mathrm{C}$.

striata Lindl

540 Calypso borealis Salisb

542 Habenaria leucostachys Watson.

$544 \mathrm{H}$. gracilis Watson.

543 Listera cordata R. Br.

547 Epipaetis gigantea Dougl.

548 Cypipedium montanum Dougl,

550 Iris maerosiphon Torr.

551 I. , tenax Dougl.

553 Sisyrinchi im bellim Watson.

$554 \mathrm{~S}$. grandiflorum Dougl.

555 Allium selioenoprasum L.

556 A. ma lidum Natson.

558 A. att muifolium: Kell.

55. Brodiaea congesta Smith.

561 B. lactea Watson.

562 B. Howelli Watson.

563 Camassia esculenti Lindi.

564 Smilacina racemosa Desf.

$565 \mathrm{~S}$ 'sessilifolia Nutt.

568 Fritillaria pudica Spreug.

569 Erythronium grandiflorum Pursh.

570 E. giranterm Lindl.

572 Calochortus Tolmiei H. \& A.

574 Prosartes Men iesii Dou.

$575 \mathrm{P}$. Oregana Watson.

577 Seoliopus Hallii Watson.

578 Trillium ovatum Pursh.

579 Stenanthium occidentale kray.

58) Zygarlenis renenosus Watson.

58t Ruppia muitima L.

5 sio Juneus Balticus Dethard.

533 J Parryi Engelm.

$591 \mathrm{~J}$. falca ns Meyer.

609 Carex Sitchensis Preseott.

611 C. Penusylvaniea fam.

62 ) Hierochloa macrophylla Thurb.

625 Phleum alpinum $\mathrm{L}$.

623 Coleanthus subtilis Seib.

63 ; Cinna pendula Trin.

63.) Descham sia elongata Presl.

640 I. - latifolia Vasey.

052 Poa confinis Vasey.

65) P. mqerantha Vas y.

$35 / \mathrm{r}$. gracilima Vasey.

660 p. nervosa Vasey.

b61 P tenuifolia Nutt.

662 Glyceria nlevata Trin.

663 G. pacitlora Presl.

670 Herdium pratense Hudson.

675 Polypodium falcatum Kell.

676 P. Scouleri Hook, \& Grey.

6.7 Gymnogramme triangularis Kf.
680 Cryptogramme acxosichoides $\mathrm{R}$. $\mathrm{Br}$.

682 Lomaria spicant Desv.

684 Phegopteris Dryopteris Tee.

685 Aspidium munitum Kf.

$1587 \mathrm{~A}$

688 A.

argutiom

690 Woodsia Oregana Eaton.

991 Lycopodiun annotinum L.

$692 \mathrm{~L}$.

elavatum $\mathrm{L}$.

694 Selaginella rupesuris Spring.

$695 \mathrm{~S}$ Douglisii spring.

$696 \mathrm{~S}$ Gregana Eaton.

6.7 Iscetes nuda kngelm.

693 I. Howellii Engelm

767 Lesquerella crcidentalis Watson.

782 Lupinus mierocarpus Lindl.

810 Saxifraga occidentalis Watson.

44 Erigeron peuciphyllus Gray.

84t5 E. Bloomeri Gray.

$746 \mathrm{z}$. corymbosus Nutt.

85. benecio hydrophyllus Nutt.

868 Gilia floceosa Gray.

93.) G. congesta Hook.

878 Hesperochiron Californieus Watson.

882 Krynitzkia cireumscissa Gray.

$833 \mathrm{~K}$ oxyearya Gray.

887 Mertensia laneeolata DC.

889 Pentstemon glaber Pursh.

872 P. deustus Dougl. var.

897 Mimulus dentatus Nutt.

902 Boschniakia strobilacea Gray.

904 Plantago pusilla Nutt.

005 Chorizanthe Watsonii T. \& G.

908 Eriogonum proliferum T. \& G.

925 Leueoerinum montanum Nutt.

926 Camassia Litehlinii Watson.

928 Fritillaria glauca Greene.

953 Bromus breviaristatus Thurb.

957 Anemone occidentalis Watson,

959 Trantvetteria grandis Nutt

960 Ranunculus Macounii Britton.

$961 \mathrm{R}$. Lyallii.

962 Myosurus lepturus.

964 Delphinium Nuttallii Gray.

967 Cardamine oceidentalis.

970 Silene Suksdorfii Robinson.

975 Trifolium microcephalum Pursh.

977 Lathyrus Nuttallii Watson.

98. Eriogynia pectinata Hook.

982 Saxifraga Tolmiei T. \& G.

933 Boykinia major Gray.

984 Bolandera Oregana Watson.

991 Boisdnvalia densiflora Watson.

1001. Erigeron Oreganus Gray.

1003 Stephanomeria minor Nutt.

1029 Iistera convallarioides Nutt.

1050 Brodiaea grandiflora Smith.

1034 Trillinm Californicum.

1038 Luzula spadicea var, melanocarpa.

1039 Carex Breweri Boott.

1049 C. stipata Mahl.

104: Heaferani Bailey.

is 4 Pleuropergen zomatnm Benth. 
1056 Anemone Uregatia Gray.

1057 Ranunculus Ratiani,

1058 R. heberarpus H. \& A.

1061 Eschscholt dia tenuifolia Hook.

1 1064 Arabis purpurascens Howeli.

1065 A. subpinnatifida Watson.

1067 Cardamine paucisecta Benth.

$1069 \mathrm{C}$ gemmata Greene.

1072 Lepidium Ureganum Howell.

1073 Thlaspi alpestre $\mathrm{L}$.

1074 Thysanocarpus radians Benth.

1075 Cleome Intea Hook.

1077 Viola occidentalis.

$1079 \mathrm{~V}$. ehrysanthe Hook.

1081 V, lobata Benth.

$1083 \mathrm{~V}$. cuneataWatson.

1084 V. Howellii Gray.

1085 Silene campanulata Watson.

1087 Cerastium angustifolium.

1103 Erodium mosehatum Willd.

1107 Rhus trilobata Nutt.

1108 R. glabra L.

1119 Astragalus Pacificus Sheldon.

$1120 \mathrm{~A}$. accidens Watson.

1121 A. umbraticus Sheldon.

1123 Lathyrus polyphyllusNutt.

1124 I. paluster var.

1125 L. Torreyi Gray.

1129 Horkelia hirsuta Lindl.

1132 Amalahchier alnifolia Nutt.

1135 Chry sosplenium glechomaefolium Nutt

1136 Tillaea minima Miers.

1142 oEnothera strigulosa T. \& G.

1145 Boisduvalia glabella Walp.

1146 Erynginm peteolatum Hook.

1147 Velaea Kelloggii C. \& R.

1149 Simm cicutaefolinm Gmelin.

1155 Cornus Canadensis L.

1165 Valerianella macrocera Gray.

1167 Enpatorium occidetale Hook.

1168 Brickellia Greenei Gray.

1169 Chrysopsis Oregana Gray.

1170 Bigelovia Douglasii Gray.

1173 Stylocline filaginea Gray.

1174 Psiloearpus tenellus Nutt.

1176 Antennaria argentea Benth.
Fraiseria Huokeriana Nutt.

is4 Blepharipappus laevis Gray.

1 1.55 Matia elegans Don.

1 iss Hemizonia Fitchii Gray.

I.110 $\mathrm{H}$ multiglandulosa Gray, var.

1146 Cotula coronopifolia L.

1200) Arnica discoidea Benth.

1202 microseris linearifolia Gray.

1209 Menziesia ferruginea Smith.

1211 Dodecatheon alpinnm Greene.

1212 Androsace septentrionalis L.

1213 Trientalis arctica Fisch.

1214 Fraxinus Oregana Nutt.

1217 Menyanthes trifoliata L.

1218 Phlox adsurgens Torr.

1229 Plagiobothry $₫$ Californica Greene.

1234 Collinsia Torreyi Gray.

1239 Pentstemon Roezli Reg.

$1240 \mathrm{P}$. heterophyllus Lindl.

1244 Mimulus luteus L.

1247 (irthocarpus lithospermoides Benth,

1250 Monardella villosa Benth.

1258 Urtica holosericea Nutt.

1261 Salix macrocarpa Nutt.

1262 Quercus Kelloggii Newberry

1268 Cephalanthera Oregana Reichenb .f.

1269 Cypripedium fasciculatum Kell.

1273 Brodiuea capitata Benth.

1274 B. Hendersoni latson.

1275 Hastingsia alba Watson.

127i; Maianthemum dilatatum.

1279 Erythronium Hendersoni Watson

$1280 \mathrm{E}$. Gitrinum Watson.

1281 E. Howellii. Watson.

1284 Trillium rivale Watson.

1285 Tofieldia occidentalis Watson.

1288 Luzula micrantha.

1298 Scirpus liniatus Michx.?

1299 Eriophorum russeolum Fries.

1:01 Carex laciniata Boott.

1303 C. multicaulis Bailey.

1304 C. polytrichoides Muhl.

1316 Gristridium australe Beauv

$1: 21$ Glyceria angustata Fries.

1329 Pellaea brachyptera Raker.

1330 Adiantum emarginatum Hook.

Clackamas Oregon,

January 14 th, 1896. 


\section{HOWELL'S PACIFIC COAST PLANTS.}

Having'spent two months botanizing in Southeastern Alaska, I now offer the following list of plants from that interesting country at the rollowing prices. Full sets, 8 ets. per specimen: less than full set 10 ets per pecimen. Yours respectfully;

Thomas Howell., CLACKAMAS, OREGON.

Dec. 10 th, 1895.

1601 Anemone narcissiflora L.

1602 Ranunculus - ? Of the occididentalis § with short eblique beak to the rotund akenes.

1603 R. ? Of the cocidentalis \&. with long straight hooked-tip beak to the oblong akenes.

1604 Kumlienia Cooleyæ Greene: Erythea ii: 193.

1605 Coptis asplenifolia Salisb. Fruit only.

1606 C. trifolia Salisb.

1607 Nasturtium = Tetrapoma Krupsianum F.\&M.

1608 Arabis hirsuta var. glabrata T. \& G.

1609 A. ambigua DC. A lyrata var.occidentalis Watson.

1610 Cochlearia Groenlandica L.?

1611 C. oblongifolia DC. ?

1612 C. officinale L. ?

1613 Viola - ?. n. sp. Near V.palustris.

1614 Stellaria brachypetala Bong.

1615 Spergularia salina Presl.

1616 Lupinus ? Like L, arcticus Watson; but the stems are branching.

1617 Rubus Chamæmorus L. Fruit only

1618 Geum ealthifolium Menzies.

1619 Poterium Sitchense Watson.

1620 P. medium,

1621 Saxifraga. The Pacific Coast representative of S. leucanthemifolia.

1622

1623

1624

1625

1626

1627

1628

1629

1630

1631

$16 \div 2$

1633

1634

1635

1636

1637

$\mathrm{S}$. punctata $\mathrm{L}$.

Epilobium glandulosum Lehm. ?

$\mathrm{E}, \quad$ Hornemanni Reichenb.?

E. adenotaulon Hausskn.? var.

Circæa alpina $\mathrm{L}$.

Selinum The American Conioselinum Fischeri.

Ligusticum Scoticum L.

?Li.

Cornus Suecica I.

Valeriana Sitchensis Bong.

Aster foliaceous Lindl.

Aster peregrinus Pursh.

Arnica intermedia n. sp.

Prenanthes alata var. sagittata Gray.

Vaccinium -? One that has been refered to a var. of V. cæspitosa.

1639

V.

? Not like any published species.

1640

V.

Chamissonis Bong.

V.

V.

n. sp. Near V. myrtilloides.

Vitis-Idaea L. 
1641 Andromedia polifolia L.

1642 Cassiope Stelleriana DC.

1643 Ledum latifolium Ait..

1644 Menziesia-_ n.sp.?

1645 Loiseleuria procumbens Desv.

1646 Cladothamnus pyrolæflorus Bong.

1647 Moneses grandiflora Gray.

1648 Dodecatheon integrifolium Bong.

1649

1650

1651

1652

1653

1654

1655

1656

1657

1658

1659

1660

1661

1662

1663

1664

D. frigidum Cham. \& Sehlecht.

Gentiana Douglasiana Bong.

G. - n. sp. Much like G. platypetala, but the corola is different.

Menyathes Crista-galli Menzies.

Castilleia parviflora Bong. Typical.

Pedicularis pedicellata Bunge.?

Boschniakia glabra C. A. Meyer.

Pinguicula vulgaris L.

Lycopus Virginicus L. A small form, 2 to 6 inches high.

Polygonum viviparum $\mathrm{L}$.

Atriplex littoralis L.

Comandra?

Iris setosa Pall.

Sisyrinchium anceps Cav.

Fritillaria Kamtschatcensis Ker.

Streptopus-? The Pacific coast S. roseus; quite different from the Eastern S. roseus Michx.

1665

1666

1667

1668

1669

1670

1671

1672

1673

1674

1675

1676

1677

1678

1679

Veratrum ? Species not determined.

Tofieldia glutinosa Willd.

Sparganium natans L.?

Potamogeton Claytoni Tuck.? A small form.

Zostera angustifolia Reichenb.

Ruppia maritima L. A small form.

Scheuchzeria palustris L.

Luzula spadicea DC.? Form with anthers equalling the filaments

I. spadicea var. subcongesta Watson.

L..

L. comosa var. congesta Watson.

L. campestris? A tall slender variety.

Juneus Balticus var. montanus Engelm.

J. filiformis L.?

J. castaneus L.? Anthers not pointed and not half as long as the filaments.

$1680 \mathrm{~J}$. nodosus L.

1681

1682

1683

1684

1685

1686

1687

1688

1689

1690

1691

J. xiphoides var. triandrus Engelm.

Eriophorum polystachyum L.

Rhynchospora alba Vahl.

Eleocharis palustris $\mathrm{R}$. Br.

E.

E.

E.

rex pauciflora Lightfoot.

C.

C.

C.

? Near C. rostrata.

, $, \quad, \quad$, 


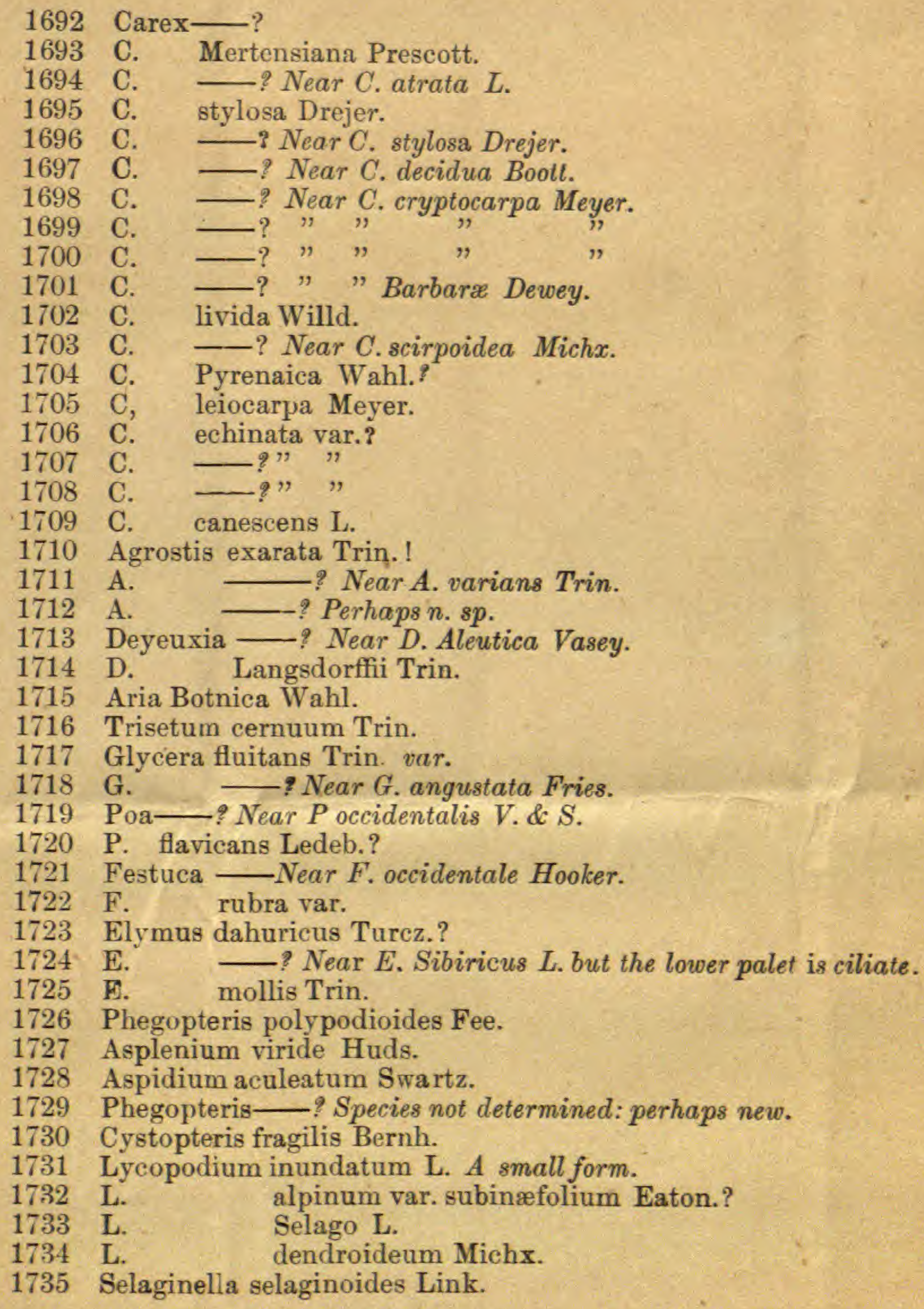

- The undetermined species will be sent to specialests for identification. 
